HEAT TRANSFER- ASIAN RESEARCH

Accepted June $12^{\text {th }} 2019$

Online ISSN:1523-1496; Publisher - Wiley

\title{
THERMAL SLIP AND RADIATIVE HEAT TRANSFER EFFECTS ON ELECTRO-OSMOTIC MAGNETO-NANOLIQUID PERISTALTIC PROPULSION THROUGH A MICROCHANNEL
}

\author{
${ }^{1}$ J. Prakash, ${ }^{2}$ E.P. Siva, ${ }^{* 3}$ D. Tripathi and ${ }^{4}$ O. Anwar Bég \\ ${ }^{I}$ Department of Mathematics, Avvaiyar Government College for Women, Karaikal-609 602, Puducherry -U.T., India \\ ${ }^{2}$ Department of Mathematics, SRM Institute of Science and Technology, Kattankulathur 603203, Tamil Nadu, India. \\ ${ }^{3}$ Department of Science and Humanities, National Institute of Technology, Uttarakhand -246174, India \\ ${ }^{4}$ Department of Mechanical/Aeronautical Engineering, Salford University, Manchester, M54WT, UK.
}

*Corresponding author: dtripathi@nituk.ac.in

\begin{abstract}
A mathematical study is described to examine the concurrent influence of thermal radiation and thermal wall slip on the dissipative magnetohydrodynamic electro-osmotic peristaltic propulsion of a viscous nano-liquid in an asymmetric microchannel under the action of an axial electric field and transverse magnetic field. Convective boundary conditions are incorporated in the model and the case of forced convection is studied i.e. thermal and species (nanoparticle volume fraction) buoyancy forces neglected. The heat source and sink effects are also included and the diffusion flux approximation is employed for radiative heat transfer. The transport model comprises the continuity, momentum, energy, nanoparticle volume fraction and electric potential equations with appropriate boundary conditions. These are simplified by negating the inertial forces and invoking the Debye-Hückel linearization. The resulting governing equations are reduced into a system of non-dimensional simultaneous ordinary differential equations, which is solved analytically. Numerical evaluation is conducted with symbolic software (MATLAB). The impact of different control parameters (Hartmann number, electroosmosis parameter, slip parameter, Helmholtz-Smoluchowski velocity, Biot numbers, Brinkman number, thermal radiation and Prandtl number) on the heat, mass and momentum characteristics (velocity, temperature, Nusselt number etc.) are presented graphically. Increasing Brinkman number is found to elevate temperature magnitudes. For positive Helmholtz-Smoluchowski velocity (reverse axial electrical field) temperature is strongly reduced whereas for negative Helmholtz-Smoluchowski velocity (aligned axial electrical field) it is significantly elevated. With increasing thermal slip nanoparticle volume fraction is also increased. Heat source elevates temperatures whereas heat sink depresses them, across the micro-channel span. Conversely, heat sink elevates nano-particle volume fraction whereas heat source decreases it. Increasing Hartmann (magnetic) parameter and Prandtl number enhance the nano-particle volume fraction. Furthermore, with increasing radiation parameter the Nusselt number is reduced at the extremities of the micro-channel whereas it is elevated at intermediate distances. The results reported provide a good insight into biomimetic energy systems exploiting electromagnetics and nanotechnology and furthermore they furnish a useful benchmark for experimental and more advanced computational multi-physics simulations.
\end{abstract}

KEYWORDS: Nanofluids; peristaltic hydrodynamics; electric double layer; electric field; magnetic field; slip boundary conditions; bio-inspired design; heat source/sink; convection; radiative heat transfer. 


\section{NOMENCLATURE}

Symbol Description

$\left(a_{1}, a_{2}\right) \quad$ dimensional (wave) amplitude of the lower and upper walls

$B_{0} \quad$ A uniform magnetic field normal to the

walls of channel ( $\bar{Y}$-axis)

c wave speed

$c^{\prime} \quad$ volumetric volume expansion coefficient

$(C, \bar{C})$

$C_{0}, C_{1}$

$D_{B}$

$D_{T}$

$\left(d_{1}+d_{2}\right)$

$e$

$E_{x}$

$\tilde{k}$

$(P, \bar{P})$

$Q_{0}$

$q$

$t^{\prime}$

$T_{0}, T_{1}$

$(T, \bar{T})$

$T$

$T_{v}$

$T_{m}$

$T_{\infty}$

$(U, V)$

$(\bar{U}, \bar{V})$ nano-particle volume fractions in the wave and fixed

frame of references

nanoparticle concentration at the lower and upper

walls respectively

Brownian diffusion coefficient

themophoretic diffusion coefficient

dimensional width of the channel

fundamental charge

applied electrical field

mean absorption coefficient

pressures in wave and fixed frame of references

heat source/sink parameter

dimensional volume flow rate in laboratory frame

dimensional time

Temperature at the lower and upper walls respectively

nano-particle temperature in the wave and fixed

frame of references

nanoparticle temperature

averaged temperature

nanofluid mean temperature

free steam temperature

velocity components in the wave frame $(X, Y)$

velocity components in the laboratory frame $(\bar{X}, \bar{Y})$

\section{Unit}

m

$\mathrm{kg} / \mathrm{s}^{2}$

$\mathrm{m} / \mathrm{s}$

$m^{3}$

$\mathrm{kg} / \mathrm{m}^{3}$

$\mathrm{kg} / \mathrm{m}^{3}$

$\mathrm{m} / \mathrm{s}$

$\mathrm{m}^{2} / \mathrm{s}$

$m$

kg. $m / s^{3} A$

$\mathrm{m}^{2} / \mathrm{kg}$

$\mathrm{kg} / \mathrm{m} . \mathrm{s}^{2}$

$\mathrm{m}^{2} / \mathrm{s}^{3}$

$\mathrm{m}^{3} / \mathrm{s}$

$s$

K

K

K

K

K

K

$\mathrm{m} / \mathrm{s}$

$m / s$ 


\section{Greek Symbols}

\begin{tabular}{|c|c|c|}
\hline $\bar{\kappa}$ & thermal conductivity & $W / m . K$ \\
\hline$\mu$ & dynamic viscosity & $\mathrm{kg} / \mathrm{m} . \mathrm{s}$ \\
\hline$\lambda$ & wave length & $m$ \\
\hline $\bar{\Phi}$ & electric potential & $\mathrm{kg} \mathrm{m}^{2} / A \mathrm{~s}^{3}$ \\
\hline$\varepsilon$ & dielectric permittivity of the medium & $A^{2} s^{4} / \mathrm{kgm}^{3}$ \\
\hline$\kappa$ & Debye length & $m$ \\
\hline$\rho_{f}$ & density of the nanofluid & $\mathrm{kg} / \mathrm{m}^{3}$ \\
\hline$\rho_{p}$ & density of the nanoparticle & $\mathrm{k} g / m^{3}$ \\
\hline$\sigma^{\prime}$ & electrical conductivity of the nanoliquid & $S / m$ \\
\hline$\tilde{\sigma}$ & Stefan-Boltzmann constant & $W / m^{2} K^{4}$ \\
\hline
\end{tabular}

\section{Dimensionless Parameters}

$(a, b) \quad$ dimensionless amplitude of the lower and upper walls

$\left(B h_{1}, B h_{2}\right) \quad$ Biot numbers at lower and upper walls

$\mathrm{Br} \quad$ Brinkmann number

$d \quad$ dimensionless width of the chennal

$\Theta \quad$ dimensionless time average flux

$\sigma \quad$ Dimensionless rescaled nanoparticle volume fraction

$\theta \quad$ dimensionless temperature

Ec Eckert number

$F \quad$ dimensionless mean flows

$k_{B} \quad$ Boltzmann constant

$q r \quad$ uni-directional thermal radiative flux

$M \quad$ Hartmann number

$N t \quad$ thermophoresis parameter

$\mathrm{Nb} \quad$ Brownian motion parameter

$\mathrm{Nu} \quad$ Nusselt number (wall heat transfer rate)

Pr Prandtl number

$p \quad$ dimensionless pressure

$R \quad$ Reynolds number

$R n \quad$ thermal radiation parameter

$(u, v) \quad$ dimensionless velocity components in the $(x, y)$ axis

Uhs Helmholtz-Smoluchowski velocity

$L \quad$ thermal slip parameter 


$\begin{array}{ll}\delta & \text { wave number } \\ \tau & \text { ratio of the heat capacity of nanoparticle material heat capacity of the nanoliquid } \\ \psi & \text { stream function } \\ \beta & \text { heat source or sink parameter } \\ n_{0} & \text { bulk concentration (number density) } \\ z & \text { valence of ions }\end{array}$

\section{INTRODUCTION}

In recent years, peristaltic fluid dynamics has garnered increasing attention due to its extensive relevance to bio-engineering science and biological propulsion. The peristaltic mechanism features in the movement of chyme in the gastrointestinal tract, transport of the ovum in female fallopian tube, dialysis devices, plasma actuation of aerodynamic flows, transport of noxious wastes in nuclear industries, excretory movement, blood pumps, heart-lung machines, vasomotion of small blood vessels, bat wing control and snake locomotion. Significant interest was stimulated in analyzing the mechanism of peristaltic propulsion largely by Latham ${ }^{[1]}$ who presented a robust engineering appraisal (both experimental and theoretical) of peristaltic flow in the human uterus frame work. This study was confined to Newtonian viscous fluids and tubular geometries. A generalized mathematical framework for modelling peristalsis as a train of sinusoidal waves in an infinitely long symmetric channel or tube was later presented by Shapiro et al. ${ }^{[2]}$ and Fung and $\mathrm{Yih}^{[3]}$. These studies generically assumed that the amplitude of the peristaltic waves is low or the wavelength of the peristaltic waves is large. Many researchers subsequently extended these studies to consider alternative geometries and increasingly more sophisticated fluids. These include Haroun $^{[4]}$ (fourth order fluids in inclined channels), Mekheimer et al ${ }^{[5]}$ (endoscopic couple stress annular flows), Hayat et al. ${ }^{[6]}$ (third grade viscoelastic peristaltic flow), Bég et al. ${ }^{[7]}$ (hydromagnetic pumping of magnetic Williamson elastic-viscous liquids), Srinivas and Kothandapani $^{[8]}$ (thermal convection in peristaltic flow), Kothandapani and Srinivas ${ }^{[9]}$ (peristaltic pumping in porous tilted channels), Hayat et al. ${ }^{[10]}$ (micropolar endoscopic flow), Vajravelu et $a l .{ }^{[11]}$ (viscoplastic peristaltic flow with peripheral Newtonian flow) and Ali et al. ${ }^{[12]}$ (Carreau shear-dependent peristaltic flow in coiled channels).

In recent years, the mechanics of nanofluids has attracted substantial interest owing to the superior characteristics of such suspensions in enhancing thermal conductivity of base fluids. Since, the pioneering work of $\mathrm{Choi}^{[13]}$, nanofluids have been explored in many applications. The research on 
nanofluids (e.g. nano-liquids) has largely concentrated on demonstrating improvements in transport characteristics and they have been deployed in biomedical systems, nuclear reactor cooling, sterilization, fuel doping, geotechnical remediation and smart drug delivery. Other notable applications include microelectronics, solid-state lighting and manufacturing, nano-porous materials for size exclusion chromatography, cell repair machines, protein engineering, active transport in ionic channels, neuro-electronic interfaces, molecular motors in biology, charge-based filtration in the kidney basal membrane and innovative nuclear systems ${ }^{[14-18]}$. In parallel with laboratory and field studies of nanofluids, a number of computational and analytical studies of nanofluids have been presented utilizing various nanoscale models (Buongiorno two-component, Pak-Cho model, Tiwari-Das volume fraction model etc). Khan and Pop ${ }^{[19]}$ investigated that the fundamental two-dimensional boundary layer flows of a nanofluid over a stretching sheet with Buongiorno's model. This work was extended by Rana and Bhargava ${ }^{[20]}$ to the case of an extending boundary (nonlinear stretching sheet) with convective boundary conditions. Mustafa et al. ${ }^{\text {[21] }}$ determined exact solutions for boundary layer nanoliquid stagnation-point flow from a stretching sheet. A subset of nanofluids is magnetite nanofluids. These have magnetic (electricallyconducting) properties which allows them to respond to applied external magnetic fields and act as smart fluids by virtue of the ferrite nano-particles. Magnetic nanofluid dynamics arises in many applications and can be simulated by amalgamating magnetohydrodynamics (MHD) with nanoscale fluid dynamics. As such magnetic nanofluids provide the double advantage of thermal enhancement and smart response in engineering systems. In recent years numerous studies of magnetic nanofluids (liquids) have been communicated. Uddin et al. ${ }^{[22]}$ investigated Falkner-Skan flows of magnetite nanofluids featuring micro-organism doping in bio-inspired materials synthesis. Shamshuddin et al. ${ }^{[23]}$ explored the cooling, anti-corrosive and tribological properties of magnetic bio-nanofluids using Boungiorno's model. Zohra et al. ${ }^{[24]}$ studied spin coating of aerospace components with magnetic nanoliquids. Bég et al. ${ }^{[25]}$ analysed numerically the load capacity characteristics of magnetic nanoliquids in orthopaedic squeezing lubrication. While magnetic nanofluids are synthesized to respond to magnetic field, electromagnetic nanofluids offer the ability to be manipulated by both electrical and magnetic fields simultaneously. Electromagnetic nanofluids have been studied in bio-polymeric coating systems by Zohra et al. ${ }^{[26]}$. Shukla et $a l .{ }^{[27]}$ presented homotopy solutions for time-dependent stagnation flows of smart nanofluids under dual action of axial electrical and transverse magnetic fields. These studies 
revealed that the momentum, thermal and species characteristics are very differently influenced by electrical and magnetic fields. Peristaltic flows of both electrically non-conducting and conducting nanofluids have also received some attention. Tripathi et al. ${ }^{[28]}$ used.

Mathematica software to compute the influence of Brownian motion, thermophoresis and buoyancy effects on nanoparticle fraction, temperature, velocity and pressure difference in a finite length nanofluid peristaltic micropump. Abbasi et al. ${ }^{[29]}$ conducted a second law thermodynamic optimization study of Hall magnetohydrodynamic peristaltic nanofluid pumping with variable viscosity using He's homotopy perturbation method, noting that increasing nano-particle volume fraction reduces entropy generation rate and decelerate the flow. Akbar et al. ${ }^{[30]}$ studied magnetic nanofluid pumping with different shape nanoparticles (bricks, platelets, cylinders), noting the modification in heat transfer characteristics with geometric shape factor.

The above studies generally neglected thermal radiation heat transfer ${ }^{[31]}$. In many biological applications, radiative heat transfer arises. These include ultra-specific thermal treatment of cells $^{[32]}$, cryopreservation through verification in biotechnology $\mathrm{y}^{[33]}$, ophthalmic treatment with radiative pulsed heating ${ }^{[34]}$ and photosynthesis (solar radiation exposure of plants) ${ }^{[35]}$. Radiative heat transfer is significantly more complex than thermal conduction and/or thermal convection since it features integro-differential formulations, spectral characteristics, wavelengths, scattering and many other phenomena. Numerical simulation of general equation of radiative heat transfer remains challenging and therefore algebraic flux models remain popular in analysis. These include the Rosseland model (diffusion approximation), Milne-Eddington approximation and the Hamaker six flux model. The Rosseland model is a diffusion approximation and relatively simple for implementation in peristaltic fluid dynamics, although it is limited to fluids of high optical thickness. Radiative properties of nanofluids have been studied both with regard to medical applications $^{[36]}$ and also energy systems (solar collectors) ${ }^{[37]}$. These studies have shown that thermal diffusivity, viscosity, thermal conductivity, convective heat transfer coefficients, and optical properties of nanofluids are all superior to conventional heat transfer fluids (base fluids). Radiative heat transfer in nanofluid flows has been examined for peristaltic pumping systems by Prakash et al. ${ }^{[38]}$ who also considered magnetic properties and viscosity variation. Mehmood et $a l .{ }^{[39]}$ used a modified Buongiorno model to analyze non-orthogonal stagnation flows of nanoliquid 
solar cell coatings with high thermal radiative flux, thermal jump and viscosity variation. Kuharat and Bég ${ }^{[40]}$ conducted computational fluid dynamic simulations of annular solar collectors doped with metallic nanofluids (Titanium oxide/Copper oxide-water) using STS and P1 radiative flux models and ANSYS finite volume software. These studies revealed that the thermal enhancement achieved with nanofluids is further accentuated with strong radiative flux conditions.

Consideration of simultaneous thermal radiation and magnetic field effects in peristaltic transport in channel/tubes features extensively in modern clinical applications including nuclear magnetic resonance diagnostic tools, thermal ablation therapy in cancer treatment, tissue repair, and ocular radiative magnetic treatment. In NMR cancer treatment both static and alternating magnetic fields and thermal radiative flux are frequently utilized and these processes involve the targeted treatment of tumor or abnormal growths in damaged tissues/cells with minimal damage to adjacent normal cells and healthy organs. It is therefore beneficial to develop mathematical models of these processes to aid medical practitioners. The effects of thermal radiation and magnetic field on peristaltic motion of Carreau nanofluids has been elaborated by Hasona et al. ${ }^{[41]}$. This study examined the influence of temperature - dependent magnetic field and considered radiotherapy and thermotherapy techniques in modern medicine. The magnetohydrodynamic and thermal radiation characteristic of a nanofluid over a vertical surface (of relevance to magnetic thermal ablation therapy) was studied by Sudarasana reddy et al. ${ }^{[42]}$ who also included the effects of a porous wall (an idealized model of human skin) and convective boundary conditions. The influence of heat and mass transfer characteristics in MHD nanoliquid transport over an inclined vertical plate was explored by Sudarsana Reddy et al. ${ }^{[43]}$. They also considered the impact of thermal radiation and heat source/sink parameter (hot spot or cold spot treatment) and observed that temperature and nano-particle concentration distributions are boosted with stronger magnetic field parameter and additionally that temperature magnitudes are ramped up with stronger radiative heat flux effect.

The science of electro-kinetics was introduced by Reuss in 1809. A sub-set of this phenomena is electro osmotic flow (EOF) or electro-osmosis. This constitutes the transport of fluid in any conduit (e.g. micro-channel, the capillary tube) under the influence of an applied external electric field. When an electric solid surface comes into contact with water, or any sedimentary solution, a negative electric field will be generated on this surface. Also, the positive and negative ions in the liquid will be under the influence of this surface. A thin layer with an 
unbalanced electric field is thereby synthesized and this is known as the electric double layer (EDL). As there is a charged field parallel to the solid surface, the non-negatively charged electric double layer (EDL) can be manipulated in the direction of the electric field. The applied electrical potential causes ion migration. Electroosmotic flow is an essential component in chemical separation techniques, notably capillary electrophoresis and can arise in natural unfiltered water as well as buffered solutions. The electro-osmotic mechanism has been successfully deployed in optimizing many devices in medicine ${ }^{[44]}$ and other areas of technology ${ }^{[45]}$. Many analytical models have also been developed for electro-osmotic flows in various configurations. Bandopadhyay et $a l .{ }^{[46]}$ described the effects of finite ionic size and polarization on the response of EDL at two cation- exclusive electrodes in non-equilibrium situations, showing that dramatic variations in the net impedance are achieved for reasonably thick EDLs as related to extremely thin ones. Tripathi et al. ${ }^{[47]}$ derived analytical solutions for electro-osmotic body force effects on capillary peristaltic hemodynamics. Tripathi et al. ${ }^{[48]}$ also examined transient Newtonian peristaltic electro-osmotic transport in a micro-channel with an integral number of waves propagating. They noted that with a reduction in EDL thickness there is a boost in the magnitude of electric potential function whereas the hydrodynamic pressure is reduced whereas the quantity of trapped boluses is enhanced. They also observed that for stronger axial electric field, there is significant axial flow deceleration and a decrease in the number of trapped boluses. Schit et al.$^{[49]}$ analyzed the impact of wall slip velocity on rotating electroosmotic flow in a gradually varying microfluidic channel. Guo and $\mathrm{Ki}^{[50]}$ investigated electro-osmotic peristaltic flow of a viscoelastic fluid through a cylindrical micro-channel with the fractional Jeffreys constitutive model and a Laplace transform technique. Schit et al. ${ }^{[51]}$ studied the electro-osmotic flow (EOF) of blood in a hydrophobic microfluidic channel under a transverse magnetic field. Prakash et al. ${ }^{[52]}$ have considered the dual influence of peristaltic pumping and electroosmosis on the flow of nanoliquid in a microfluidic channel. Tripathi et al. ${ }^{[53]}$ examined the influence of different peristaltic wave forms, thermal and solutal buoyancy and Joule electrical dissipation on electro-osmotic peristaltic motion of aqueous nano-liquids in a microfluidic channel. Prakash et al. ${ }^{[54]}$ have studied radiative heat transfer effects in peristaltic motion of micropolar nanofluid flow altered by electroosmosis in a microfluidic vessel using Matlab bvp4c subroutines.

In the current study, a mathematical model is developed to simulate the magnetohydrodynamic electroosmosis-modulated peristaltic pumping of viscous nanofluids in a 
micro-fluidic asymmetric channel. An axial electrical field and transverse magnetic field are imposed. Convective boundary conditions are included and forced convection is considered. Heat generation (source) and absorption (sink) effects are also included and the Rosseland diffusion flux approximation is employed for radiative heat transfer. The present analysis is confined to large wavelength and low Reynolds number assumptions and Debye-Hückel linearization. Closed form solutions are developed for stream function, nanoparticle temperature and nanoparticle volumetric concentration distributions. A parametric analysis of the transport characteristics is conducted with the aid of graphs for the influence of Hartmann (magnetic body force) number, thermal slip parameter, Helmholtz-Smoluchowski velocity, Biot numbers, Brinkman number, thermal radiation parameter and Prandtl number. The current study has thus far not appeared in the literature and constitutes a significant extension to the existing body of literature, specifically, with regard to, the combined effects of radiative heat flux, heat source/sink and thermal slip. It is envisaged that the current simulations will provide further insight into possible bio-inspired energy systems and radiation treatment of biological processes ${ }^{[55-57]}$.

\section{MATHEMATICAL MODEL}

\subsection{Geometry description}

The physical model for wall-induced motion of ionic nanofluids through an asymmetric microchannel having width $\left(d_{1}+d_{2}\right)$ in the presence of electro-magneto-hydro-dynamics (EMHD) is depicted in Fig.1. Peristaltic motion is generated by a succession of sinusoidal waves progressing along the deformable microchannel walls with constant velocity $c$ in the $\bar{X}$ direction. A uniform magnetic field of strength $B_{0}$ is imposed to normal to the walls of channel ( $\bar{Y}$-axis). The magnetic field is sufficiently weak to avoid Hall current generation and magnetic induction effects are also negated. An axial electric field is also applied and this drives the electroosmotic flow along the asymmetric microchannel. The temperature $(\bar{T})$ and concentration $(\bar{C})$ are considered as $T_{0}, C_{0}$ and $T_{1}, C_{1}$ at the lower wall $\left(\bar{H}_{1}\right)$ and upper wall $\left(\bar{H}_{2}\right)$, respectively. The deformable microchannel walls equations are considered to obey the following relations:

$$
\bar{H}_{1}=-d_{1}-a_{1} \cos ^{2}\left(\frac{\pi}{\lambda}\left(\bar{X}-c t^{\prime}\right)+\phi\right),
$$




$$
\bar{H}_{2}=d_{2}+a_{2} \cos ^{2}\left(\frac{\pi}{\lambda}\left(\bar{X}-c t^{\prime}\right)\right) .
$$

where $c, \lambda, t^{\prime}, \phi, a_{1}$ and $a_{2}$ are the wave velocity, wave-length, dimensional time, phase difference and amplitudes of the lower and upper walls respectively.

\subsection{Governing equations}

For an incompressible viscous electromagnetic nanofluid the balance equations for mass, momentum, energy (heat) and nanoparticle volumetric fraction (concentration) are given by ${ }^{[51-54]}$ :

$$
\begin{aligned}
& \frac{\partial \bar{U}}{\partial \bar{X}}+\frac{\partial \bar{V}}{\partial \bar{Y}}=0 \\
& \rho_{f}\left[\frac{\partial \bar{U}}{\partial t^{\prime}}+\bar{U} \frac{\partial \bar{U}}{\partial \bar{X}}+\bar{V} \frac{\partial \bar{U}}{\partial \bar{Y}}\right]=-\frac{\partial \bar{P}}{\partial \bar{X}}+\mu\left[\frac{\partial^{2} \bar{U}}{\partial \bar{X}^{2}}+\frac{\partial^{2} \bar{U}}{\partial \bar{Y}^{2}}\right]-\sigma^{\prime} B_{0}^{2} \bar{U}+\bar{\rho}_{e} E_{x} \\
& \rho_{f}\left[\frac{\partial \bar{V}}{\partial t^{\prime}}+\bar{U} \frac{\partial \bar{V}}{\partial \bar{X}}+\bar{V} \frac{\partial \bar{V}}{\partial \bar{Y}}\right]=-\frac{\partial \bar{P}}{\partial \bar{Y}}+\mu\left[\frac{\partial^{2} \bar{V}}{\partial \bar{X}^{2}}+\frac{\partial^{2} \bar{V}}{\partial \bar{Y}^{2}}\right], \\
& \left(\rho c^{\prime}\right)_{f}\left[\frac{\partial \bar{T}}{\partial t^{\prime}}+\bar{U} \frac{\partial \bar{T}}{\partial \bar{X}}+\bar{V} \frac{\partial \bar{T}}{\partial \bar{Y}}\right]=\bar{\kappa}\left[\frac{\partial^{2} \bar{T}}{\partial \bar{X}^{2}}+\frac{\partial^{2} \bar{T}}{\partial \bar{Y}^{2}}\right]-\frac{\partial \bar{q}_{r}}{\partial \bar{Y}}+\mu\left[4\left(\frac{\partial \bar{U}}{\partial \bar{X}}\right)^{2}+\left(\frac{\partial \bar{V}}{\partial \bar{X}}+\frac{\partial \bar{U}}{\partial \bar{Y}}\right)^{2}\right]+Q_{0} \\
& +\left[\frac{\partial \bar{C}}{\partial t^{\prime}}+\bar{U} \frac{\partial \bar{C}}{\partial \bar{X}}+\bar{V} \frac{\partial \bar{C}}{\partial \bar{Y}}\right]=D_{B}\left[\frac{\partial^{2} \bar{C}}{\partial \bar{X}^{2}}+\frac{\partial^{2} \bar{C}}{\partial \bar{Y}^{2}}\right]+\frac{D_{T}}{T_{m}}\left[\frac{\partial^{2} \bar{T}}{\partial \bar{X}^{2}}+\frac{\partial^{2} \bar{T}}{\partial \bar{Y}^{2}}\right] \cdot
\end{aligned}
$$

where $\bar{U}, \bar{V}$ are the components of velocity along $\bar{X}$ and $\bar{Y}$ directions respectively, $t^{\prime}, c^{\prime}, d / d t^{\prime}$, $\overline{\boldsymbol{P}}, \sigma^{\prime}, \rho_{f}, \rho_{p}, B_{0}, \bar{\kappa}, Q_{0}, D_{B}, D_{T}, T_{m}$ and $\tau$ are the dimensional time, volumetric volume expansion coefficient, material time derivative, pressure, electrical conductivity of the nanoliquid, density of the nanoliquid, density of the nanoparticles, uniform applied magnetic field, thermal conductivity, heat generation/absorption, Brownian diffusion coefficient, thermophoretic diffusion coefficient, nanoliquid mean temperature, ratio of the effective heat capacity of nanoparticle material and heat capacity of the nanoliquid. Uni-directional thermal radiative flux $\left(q_{r}\right)$ is present. Assuming high optical thickness and absorptivity of the nanoliquid and neglecting non-gray and scattering effects, the Rosseland diffusion approximation for radiative heat flux is employed, for 
which the radiative flux is uni-directional, of the form, $\bar{q}_{r}=-\frac{4 \tilde{\sigma}}{3 \tilde{k}} \frac{\partial \bar{T}^{4}}{\partial \bar{Y}}$, and circumvents the need to solve the integro-differential radiative heat transfer equation. The temperature difference within the fluid flow is sufficiently small such that the term $\bar{T}^{4}$ may be expressed as linear function in a Taylor series about a free stream temperature $T_{\infty}$ via neglecting higher order terms in the first order in $\left(\bar{T}-T_{\infty}\right)$, we get:

$$
\bar{q}_{r}=-\frac{16 \tilde{\sigma} T_{\infty}^{3}}{3 \tilde{k}} \frac{\partial \bar{T}}{\partial \bar{Y}}
$$

Here $\tilde{k}$ and $\tilde{\sigma}$ are the mean absorption coefficient and Stefan-Boltzmann constant respectively. We further note that the Rosseland radiation model makes the assumption that the intensity is the black-body intensity at the fluid temperature.

\subsection{Electrohydrodynamics}

The well-known Poisson equation is defined as ${ }^{[47,48]}$ :

$$
\nabla^{2} \bar{\Phi}=-\frac{\bar{\rho}_{e}}{\varepsilon}
$$

where $\bar{\Phi}$ is the electric potential and $\varepsilon$ is the dielectric permittivity of the medium. The electric charge density follows the Boltzmann distribution which is specified by:

$$
\bar{\rho}_{e}=-2 n_{0} e z \sinh \left(\frac{e z \bar{\Phi}}{k_{B} T_{v}}\right)
$$

where $n_{0}, T_{v}, z, e$ and $k_{B}$ represent the bulk concentration (number density), averaged temperature, valence of ions, elementary charge valence and Boltzmann constant, respectively.

\subsection{Dimensional analysis and approximations}

Introducing a wave frame $(X, Y)$ moving with velocity $c$ away from the fixed frame $(\bar{X}, \bar{Y})$, the following transformations may be applied:

$$
X=\bar{X}-c t^{\prime}, Y=\bar{Y}, U=\bar{U}-c, V=\bar{V}, P=\bar{P}, T=\bar{T}, C=\bar{C}
$$


where $(U, V)$ and $(\bar{U}, \bar{V})$ are velocity components, $P$ and $\bar{P}$ are pressures, $T$ and $\bar{T}$ are temperatures and $C$ and $\bar{C}$ are nano-particle volume fractions (solute concentrations) in the wave (laboratory) and fixed frame of references respectively. Also, to scale the physics, it is useful to invoke the following non-dimensional quantities in Eqns. (1-7):

$$
\begin{aligned}
& x=\frac{X}{\lambda}, y=\frac{Y}{d_{2}}, u=\frac{U}{c}, v=\frac{V}{c}, \delta=\frac{d_{2}}{\lambda}, h_{1}=\frac{H_{1}}{d_{2}}, h_{2}=\frac{H_{2}}{d_{2}}, p=\frac{d_{2}^{2} P}{c \lambda \mu}, S c=\frac{v}{D_{B}}, \bar{\Phi}=\frac{\Phi}{\zeta}, \\
& \theta=\frac{T-T_{0}}{T_{1}-T_{0}}, a=\frac{a_{1}}{d_{2}}, b=\frac{a_{2}}{d_{2}}, \sigma=\frac{C-C_{0}}{C_{1}-C_{0}}, R=\frac{\rho_{f} c d_{2}}{\mu}, N b=\frac{\tau D_{B}\left(C_{1}-C_{0}\right)}{v}, N t=\frac{\tau D_{T}\left(T_{1}-T_{0}\right)}{T_{m} v}, \\
& \text { Uhs }=-\frac{E_{x} \varepsilon \xi}{\mu c}, \beta=\frac{Q_{0} d_{2}^{2}}{\left(T_{1}-T_{0}\right) \nu c_{p}}, E c=\frac{c^{2}}{c_{f} T_{m}}, M=\sqrt{\frac{\sigma^{\prime}}{\mu}} d_{2} B_{0}, \operatorname{Pr}=\frac{\mu c_{f}}{\bar{\kappa}}, R n=\frac{16 \tilde{\sigma} T_{\infty}^{3}}{3 \tilde{k} \mu c_{f}} .
\end{aligned}
$$

Utilizing Debye-Hückel linearization, the Poisson-Boltzmann equation reduces Eqn. (11) into:

$$
\frac{\partial^{2} \Phi}{\partial y^{2}}=\kappa^{2} \Phi
$$

Further, applying the lubrication approximations and introducing a dimensional stream function of the form $u=\frac{\partial \psi}{\partial y}$ and $v=-\delta \frac{\partial \psi}{\partial y}$, the governing equations contract to:

$$
\begin{aligned}
& \frac{\partial p}{\partial x}=\frac{\partial^{3} \psi}{\partial y^{3}}-M^{2}\left(\frac{\partial \psi}{\partial y}+1\right)+U h s \kappa^{2} \Phi, \\
& \frac{\partial p}{\partial y}=0
\end{aligned}
$$

$\frac{\partial^{2} \theta}{\partial y^{2}}+\left(\frac{B r}{1+R n \operatorname{Pr}}\right)\left(\frac{\partial^{2} \psi}{\partial y^{2}}\right)^{2}+\left(\frac{N b \operatorname{Pr}}{1+R n \operatorname{Pr}}\right)\left(\frac{\partial \sigma}{\partial y} \frac{\partial \theta}{\partial y}\right)+\left(\frac{N t \operatorname{Pr}}{1+R n \operatorname{Pr}}\right)\left(\frac{\partial \theta}{\partial y}\right)^{2}+\left(\frac{\beta \operatorname{Pr}}{1+R n \operatorname{Pr}}\right)=0$

$\frac{\partial^{2} \sigma}{\partial y^{2}}+\frac{N t}{N b} \frac{\partial^{2} \theta}{\partial y^{2}}=0$

Eqn. (16) shows that $p$ is an independent of $x$ and eradication of pressure from Eqns. (15-16) which are automatically satisfied by continuity, gives: 


$$
\frac{\partial^{4} \psi}{\partial y^{4}}-M^{2} \frac{\partial^{2} \psi}{\partial y^{2}}+U h s \kappa^{2} \frac{\partial \Phi}{\partial y}=0
$$

In the above equations, $p, R, a, b, \psi, \theta, \sigma, \beta, M, N b, \operatorname{Pr}, N t, E c, B r(=\operatorname{Pr} E c), R n$ are the non-dimensional pressure, Reynolds number, amplitudes of lower wall and amplitudes of upper wall, stream function, dimensionless nanoparticle temperature, dimensionless nanoparticle volumetric concentration (or rescaled nanoparticle volume fraction), non-dimensional heat source/sink parameter, Hartmann magnetic number, Brownian motion parameter, Prandtl number, thermophoresis parameter, Eckert number, Brinkman number and thermal radiation parameter.

Also in Eqn. (18), $\quad \kappa=d_{2} e z \sqrt{\frac{2 n_{0}}{\varepsilon k_{B} T_{v}}}$ is known as the electro-osmotic parameter (inverse of the EDL thickness) or Debye-Hückel parameter.

\subsection{Thermal slip boundary conditions}

The non-dimensional boundary conditions (slip and convective boundary conditions) are employed as:

$$
\begin{aligned}
& \psi=\frac{F}{2}, \frac{\partial \psi}{\partial y}+L \frac{\partial^{2} \psi}{\partial y^{2}}=-1, \frac{\partial \theta}{\partial y}=B h_{2}(1-\theta), \sigma=1 \text { and } \Phi=1 \text { at } y=h_{2}, \\
& \psi=-\frac{F}{2}, \frac{\partial \psi}{\partial y}-L \frac{\partial^{2} \psi}{\partial y^{2}}=-1, \frac{\partial \theta}{\partial y}=B h_{1} \theta, \sigma=0 \text { and } \Phi=0 \text { at } y=h_{1} .
\end{aligned}
$$

Here, $B h_{1}=\frac{h_{h_{1}} d_{2}}{k_{h}}$ and $B h_{2}=\frac{h_{h_{2}} d_{2}}{k_{h}}$ are Biot number at the lower and upper micro-channel walls respectively and $L=\frac{\sqrt{k^{\prime}}}{d_{2} \alpha^{\prime}}$ is the thermal slip parameter.

\subsection{Volumetric flow rate analysis}

The volumetric flow rate in the wave frame is given by:

$q=\int_{h_{1}}^{h_{2}} U d y=\int_{h_{1}}^{h_{2}}(\bar{U}-c) d y$,

which, on integration, yields: 
$q=F-c\left(h_{2}-h_{1}\right)$

Mean volumetric flow rate over one time period takes the form:

$$
\Theta=\int_{0}^{1} F d t=\int_{0}^{1}\left(q+c\left(h_{2}-h_{1}\right)\right) d t
$$

Integrating the above equation yields:

$$
\Theta=F+1+d+\left(\frac{a+b}{2}\right)
$$

inwhich

$$
F=\int_{h_{1}}^{h_{2}} \frac{\partial \psi}{\partial y} d y
$$

\subsection{Solution of the Problem}

Solving Eqns. (13-18) subject to the boundary conditions in Eqns. (19a, b), yields the following expressions:

Solution for stream function is:

$\psi(y)=c_{1}+c_{2} y+c_{3} e^{M y}+c_{4} e^{-M y}+N_{2} e^{-\kappa y}+N_{3} e^{\kappa y}$

Solution for axial velocity is:

$u(y)=c_{2}+M c_{3} e^{M y}-M c_{4} e^{-M y}-\kappa N_{2} e^{-\kappa y}+\kappa N_{3} e^{\kappa y}$,

Solution for temperature distribution is:

$$
\begin{aligned}
\theta(y)= & c_{5}+c_{6} e^{M y}+N_{24} e^{2 M y}+N_{25} e^{-2 M y}+N_{26} e^{-2 \kappa y}+N_{27} e^{2 \kappa y}+N_{28} y \\
& +N_{29} e^{(M-\kappa) y}+N_{30} e^{(M+\kappa) y}+N_{31} e^{-(M+\kappa) y}+N_{32} e^{(\kappa-M) y}
\end{aligned}
$$

Solution for nanoparticle volumetric concentration is :

$$
\sigma(y)=-\frac{N t}{N b}\left(\begin{array}{c}
c_{5}+c_{6} e^{M y}+N_{24} e^{2 M y}+N_{25} e^{-2 M y}+N_{26} e^{-2 \kappa y}+N_{27} e^{2 \kappa y}+N_{28} y \\
+N_{29} e^{(M-\kappa) y}+N_{30} e^{(M+\kappa) y}+N_{31} e^{-(M+\kappa) y}+N_{32} e^{(\kappa-M) y}
\end{array}\right)+c_{7} y+c_{8}
$$


The expressions for the Nusselt number (wall heat transfer rate) at the lower wall are given by:

$$
N u=\left(\frac{\partial h_{1}}{\partial x}\right)\left(\frac{\partial \theta}{\partial y}\right)_{y=h_{1}}
$$

Constants in the above expressions are provided in the Appendix section.

\section{COMPUTATIONAL RESULTS AND PHYSICAL INTERPRETATION}

Based on the closed-form solutions developed in Section 2, numerical evaluations have been conducted in symbolic software (Matlab). In this section therefore the graphical distributions for various transport characteristics (axial velocity, temperature, nanoparticle volume fraction and Nusselt number) are therefore presented in Figs. 2-5. In the simulations presented, pertinent ranges of dimensionless parameters have been adopted based on viable clinical sources. Magnetic and nanoscale parameter ranges are consistent with the suggestions of Arruebo et al. [58] and Funk et al. [59]. Electrokinetic parameters are based on the recommendations of Min et al. [60] and Bourouina et al. [61] and apply to micro-pump electro-osmotic designs. Peristaltic wave characteristics prescribed are consistent with the data and dimensionless parameter ranges specified in Bourouina et al. [61] and Busek et al. [62]. All physiological fluid dynamic and mass diffusion characteristics are based on data provided in the excellent treatise by Lightfoot et al. [63]. Finally all thermal parameters are consistent with those adopted in magnetic thermal therapy applications in Moros [64]. The parameters utilized in the present article are therefore as realistic as possible for practical biomedical and bio-energetics applications.

Figs. 2 (a-e) show the behavior of Hartmann number $(M)$, Helmholtz-Smoluchowski velocity $(U h s)$, Debye-Hückel parameter $(\kappa)$, zeta and thermal slip parameter $(L)$ on the axial velocity distribution at $x=0.5$ (intermediate distance along the channel) and $t=0.2$ (intermediate time). The parabolic nature of axial velocity evolution across the micro-channel $(-1<y<1)$ is clearly observed. The impact of Hartmann number $(M)$ on axial velocity distribution is drawn in Fig. 2(a), and it is evident that axial velocity profile reduces with enhancing Hartman number in the core region of the micro-channel. The nanoliquid is therefore decelerated substantially about the centreline i.e. nanoliquid velocity reduces for $y \in(-0.581,1.995) \cup(0.481,1.999)$. However the opposite effect is induced in the periphery of the micro-channel and this is attributable to a redistribution in momentum (axial flow is accelerated external to the core zone). However, the 
acceleration in the peripheral zones is less significant than the retardation in the core region. Furthermore, flow reversal is never caused in the core region. This result is characteristic of magnetohydrodynamics since the magnetic field acts transverse to the flow direction and the associated Lorentzian magnetic drag which acts axially, retards the flow in the core region. Hence, the nanofluid axial velocity can be damped in the core region by the practical application of a static magnetic field on the flow. For the case of $M=1$ the magnetic Lorentz force and viscous hydrodynamic force are equal. For $M>1$ magnetic drag dominates. The influence of HelmholtzSmoluchowski velocity ( $U h s)$ on axial velocity $(u)$ distribution is given in Fig. 2(b). It is concluded that axial velocity reduces for $y \in(0.008,1.542)$ with the changes in the magnitude of HelmholtzSmoluchowski (both positive and negative values) on velocity profile $(u)$. The opposite condition is observed in the remainder of the microchannel span range. Effectively negative Uhs (which corresponds to positively orientated axial electrical field) accelerates the flow in the lower microchannel half space whereas positive $U h s$ (which corresponds to reversely orientated axial electrical field) induces deceleration. These trends are reversed in the upper micro-channel half space. The direction of electrical field and the location within the micro-channel are therefore key factors contributing to the nature of the velocity distribution. The response of velocity profile to DebyeHückel parameter $(\kappa)$ is visualized in Fig. 2(c). It is apparent that increasing Debye-Hückel parameter $(\kappa)$ i.e. decreasing EDL thickness, clearly decelerates the axial flow in the lower microchannel space whereas it manifests in acceleration in the upper micro-channel space. The influence of thermal slip parameter $(L)$ on axial velocity distribution as plotted in Fig. 2(d) shows the opposite behavior to that produced with increasing Hartmann number. Maximum axial velocity is obtained with zero thermal slip and the magnitude is strongly reduced in the core zone with increasing thermal slip. However, in the peripheral region, there is a strong acceleration in the flow and the effect is maximum at the micro-channel walls (where thermal jump is imposed). It is also pertinent to note that while thermal jump boosts the axial velocity at the micro-channel walls, the overall maximum axial velocity for the no-slip case $(L=0)$ significantly exceeds the peaks at the walls even with strong thermal slip.

Figs.3-4 illustrate the temperature distribution and nanoparticle volume fraction with various values of the heat transfer Biot number $\left(B h_{1}\right)$, slip parameter $(L)$, Brinkman number $(B r)$, 
Hartmann number $(M)$, Prandtl number $(\operatorname{Pr})$, Helmholtz-Smoluchowski velocity $(U h s)$ for fixed values of heat source $(\beta=2)$ and heat $\operatorname{sink}(\beta=-2)$. The influence of heat transfer (thermal) Biot number at the lower wall $\left(B h_{1}=\frac{h_{h_{1}} d_{2}}{k_{h}}\right)$ on the temperature distribution is depicted in Fig.3(a) for negative and non-negative values of heat source/sink parameter $(\beta=-2 \& 2)$. It is clear that increasing thermal Biot number corresponds to a decrease in the thermal conductivity of the nanofluid and this results in a reduction in temperatures of the regime for both heat source and sink parameter cases. However consistently higher temperatures are generated for the case of heat source $(\beta=2)$ as compared with the case for heat sink $(\beta=-2)$. Internal heat generation, which may arise in for example spot thermal therapy in biomedical treatments, clearly elevates the temperatures across the micro-channel span. The converse effect is generated with a heat sink which is suitable for cooling. Furthermore, significantly higher temperatures are generated at the lower wall, associated with the thermal Biot number imposed there i.e. $\left(B h_{1}=\frac{h_{h_{1}} d_{2}}{k_{h}}\right)$ compared with the upper wall. Fig. 3(b) presents the distribution of temperature against $y$ for different values of slip parameter $\left(L=\frac{\sqrt{k^{\prime}}}{d_{2} \alpha^{\prime}}\right)$ for both heat source and heat sink scenarios i.e. $\beta=-2 \& 2$. Clearly increasing thermal jump (slip), which is proportional to thermal conductivity, decreases the temperature across the entire width of the microfluidic channel. Greater quantities of heat are transferred from the nanoliquid body to the micro-channel walls i.e. thermal diffusion is elevated to the walls is elevated with increasing thermal slip. This serves to cool the nanoliquid regime. Similar observations have been reported by Zohra et al. ${ }^{[24]}$ and Mehmood et al. ${ }^{[39]}$. A more homogenous temperature distribution is computed at both walls at any value of thermal slip parameter, as compared with the profiles associated with the variation in the lower wall thermal Biot number described earlier. Fig. 3(c) presents the evolution in nanoliquid temperature with a modification in Brinkman number i.e. $\operatorname{Br}(=\operatorname{Pr} E c)$ again for both cases of heat source $(\beta=2)$ and heat sink $(\beta=-2)$. Three different values of Brinkman number are studied i.e. $B r=0,0.25$, 0.5 and it is evident that greater Brinkman number strongly enhances temperature magnitudes. Brinkman number defines the ratio of heat produced by viscous dissipation and heat transported 
by molecular conduction. When $B r=1$, the viscous heating relative to heat conduction can be ignored in the micro-channel. For large values of Brinkman number, the kinetic energy dissipated in the viscous ionic nanoliquid exerts a substantial influence on the temperature distribution. Larger Brinkman numbers imply a greater quantity of energy conversion to heat which results in temperature enhancement in the micro-channel. It is also interesting to note that again heat source $(\beta=2)$ increases temperatures and the opposite effect is induced with heat $\operatorname{sink}(\beta=-2)$. However, for both sets of profiles the Brinkman number has the same effect. Furthermore, the profiles for the former case $(\beta=2)$ are inverted parabolas across the span whereas in the latter case $(\beta=-2)$ they are parabolas. Fig.3(d) illustrate the temperature profile for different values of Hartmann number $(M)$ with a heat sink and heat source present $(\beta=-2 \& 2)$. For the case of heat source present, an increase in Hartmann number leads to a reduction in nanoliquid temperature magnitudes. However, the opposite effect is generated when a heat sink is present. The dissipation of work expended in dragging the nanoliquid against the action of magnetic field is elevates with greater Hartmann number and this increases temperatures, for the case of a heat sink. Weaker magnetic field leads to lower temperatures for the case of a heat sink but higher temperatures for the case of a heat source. Overall the heat source/sink effect is more critical to the temperature distribution than the magnetic field imposed. This may be exploited for example in the administration of magnetic nano-particles in cancer therapy via electro-osmotic diffusion in the blood. Fig.3(e) shows the influence of Prandtl number $(\operatorname{Pr})$ on fluid temperature distribution $\theta$ across the micro-channel span again for two different values of heat source/sink parameter. The Prandtl number is selected as $\operatorname{Pr}=0.044,0.71$ and 7 , corresponding to liquid metal (mercury), air and water respectively, all of which are common base fluids in energy and biomedical applications. It is observed that increasing Prandtl number $(\operatorname{Pr})$ leads to an increase in the nanoliquid temperature $(\theta)$ with a heat source whereas it results in a decrease with a sink parameter. The parameter $\mathrm{Pr}$ is the most important dimensionless number in heat transfer. It defines the ratio of momentum diffusion to the thermal diffusion. For $\operatorname{Pr}>1$, momentum diffusion dominates the heat diffusion and vice versa for $\operatorname{Pr}<1$. Higher $\operatorname{Pr}$ values imply a lower thermal conductivity of the nanoliquid. As $\operatorname{Pr}$ is the only non-dimensional parameter that categorizes thermofluid properties, $\operatorname{Pr}$ should be varied in order to generalize the solutions to different types of base liquids. With 
greater $\operatorname{Pr}$, thermal conductivity is strongly affected and heat transfer is enhanced for the case of a heat source. The converse trend is observed for a heat sink where lower Prandtl number elevates the temperatures. Fig. 3(f ) shows the temperature profile on different values of HelmholtzSmoluchowski velocity $(U h s)$. For positive $U h s$ velocity (reverse axial electrical field) temperature is strongly reduced whereas for negative Uhs (aligned axial electrical field) it is significantly elevated. The case of vanishing electrical field $(U h s=0)$ lies between the other two cases. Clearly the direction of axial electrical field significantly modifies the temperature field. Cooling is associated with reverse electrical field whereas heating of the ionic nanoliquid is generated with aligned axial electrical field.

Figs. 4(a-b) are plotted to determine the influence of lower wall thermal Biot number $\left(B \boldsymbol{h}_{1}\right)$ and thermal slip $(\boldsymbol{L})$ on the nanoparticle volume fraction with heat source/sink cases. Nanoparticle volume fraction reduces between the micro-channel boundaries with increasing $B h_{1}$ as observed in Fig. 4a. Significantly higher magnitudes are computed at the upper micro-channel wall $(y=1)$ compared with the lower micro-channel wall $(y=-1)$. Heat sink $(\beta<0)$ produces considerably greater magnitudes of nanoparticle volume fraction compared with heat source $(\beta>0)$ across the entire micro-channel span. Fig 4b shows that the conversely with increasing thermal slip at the micro-channel walls, there is an enhancement in the nanoparticle volume fraction. However, the heat sink produces consistently higher nanoparticle volume fraction magnitudes than the heat source. Also as in Fig 4a, markedly greater magnitudes of nanoparticle volume fraction are computed at the upper micro-channel wall $(y=1)$ compared with the lower wall $(y=-1)$ i.e. there is invariably an ascent in nanoparticle volume fraction profiles from the lower wall to the upper wall, irrespective of the heat source/sink or thermal slip parameter prescribed. The exacerbation in thermal conduction may contribute to the enhanced interaction of nano-particles and the boost in magnitudes in nanoparticle volume fraction. Clearly thermal Biot number and thermal slip exert a very different influence on temperature and nano-particle (species) distributions. However, there will inevitably be a contribution from Brownian motion and also thermophoretic body force, which although not studied explicitly, are still present in the magnetic electro-osmotic nanofluid peristaltic regime. Convective boundary and thermal jump conditions are therefore shown to induce non-trivial effects and their neglection can lead to incorrect predictions in both heat and 
mass (nano-particle) species diffusion in ionic nanoliquids. In Figs.4 (c-f) the evolution of nanoparticle volume fraction $(\sigma)$ with respectively Brinkman number $(B r)$, Hartmann number $(M)$, Prandtl number (Pr) and Helmholtz-Smoluchowski velocity $(U h s)$ are presented. It is found that nanoparticle volume fraction is generally depleted with greater Brinkman number i.e. increasing viscous heating relative to thermal conduction (Fig. 4c). However, heat sink (absorption) produces a substantial elevation in nano-particle volume fraction as compared with heat source (generation). This indicates that the strategic deployment of spot heating/cooling in electro-osmotic nanofluid pumping can significantly manipulate nano-particle diffusion characteristics and this may be of benefit in targeted drug-delivery systems. Stronger magnetic field (higher Hartmann number) is observed to generally elevate nano-particle volume fraction (Fig. 4d) and this effect is maximized for the heat sink case, although it is still considerable for the heat source case. The retardation induced in the ionic nanofluid by the increasing magnitude of Lorentzian drag force opposes momentum development (principally in the core flow). The momentum diffusion rate is decreased and the thermal and species diffusion rates are increased. Nano-particle diffusion is therefore encouraged with stronger magnetic field and with electrical field fixed, this may be exploited in targeted magnetic nano-carriers in pharmacodynamics ${ }^{[58]}$. However biochemical dissolution characteristics of magnetic nano-particles require a more sophisticated model for these nanostructured functional biomaterials than that presented here and this warrants further attention from researchers. With increasing Prandtl number (Fig. 4e) there is a significant elevation in nanoparticle volume fraction. Furthermore nano-particle volume fraction values are higher for heat sink as compared with heat source, in particular, at higher Prandtl number. A reduction in thermal diffusivity (higher Prandtl number) is therefore assistive to diffusion of nano-particles in the flow. Fig. 4f shows that with positive $U h s$ (reverse axial electrical field) there is a marginal increase in magnitudes of nano-particle volume fraction whereas with negative $U h s$ (aligned axial electrical field) there is a weak decrease. Heat sink $(\beta=-2)$ also generates invariably greater nano-particle volume fraction values than heat source $(\beta=2)$. The modification in electrical field direction influences primarily the momentum diffusion and also to a secondary extent the thermal diffusion. This in turn indirectly influences the nano-particle species diffusion characteristics via coupling terms between the temperature and nano-particle volume fraction fields, as exemplified by the 
term, $\frac{N b P r}{1+R_{n} P r}\left(\frac{\partial \sigma}{\partial y} \frac{\partial \theta}{\partial y}\right)$, in the energy conservation Eqn. (16) and the term, $\frac{N t}{N b} \frac{\partial^{2} \theta}{\partial y^{2}}$, in the nanoliquid species conservation Eqn. (17).

Figs. 5(a)-(g) visualize the impact of thermal Biot number, slip parameter, Brinkman number, Hartman number, thermal radiation parameter, Helmholtz-Smoluchowski velocity and heat source/sink parameter on Nusselt number distributions along the micro-channel walls. The Nusselt number $(\mathrm{Nu})$ is a dimensionless heat transfer coefficient which represents the relative contribution of heat transfer due to thermal convection to that due to thermal conduction. It allows an estimate of heat transfer rate at the micro-channel walls. For $N u=0$, the electro-osmotic peristaltic nanofluid motion is stationary and all heat transfer is by conduction and for $N u \neq 0$, the fluid motion reinforces heat transfer by advection. The peristaltic motion (contraction and expansion of the walls) is generated by sinusoidal waves propagating along the micro-channel walls. This leads to an oscillatory behavior in wall characteristics and explains the undulating profiles of Nusselt number in Figs. 5a-g. The effect of heat transfer Biot number on Nusselt number of the nanofluid is considered in Fig. 5(a). It is apparent that the absolute value of Nusselt number is generally enhanced with increasing value of thermal Biot number at lower wall $\left(B h_{1}=\frac{h_{h_{1}} d_{2}}{k_{h}}\right)$. Temperature gradient is enhanced with decreasing thermal conductivity of the nanofluid (higher thermal Biot number) and this elevates the Nusselt number values at the walls. Conversely (Fig. $5 b)$ increasing thermal slip parameter is observed to reduce the Nusselt number, both at the entry and exit locations of the micro-channel (i.e. at $x=0, x=2$ respectively) and also at intermediate distance along the micro-channel. The elevation in temperature in the body of the ionic nanoliquid (computed in earlier graphs) is due to reduced thermal energy transfer to the wall with thermal jump increasing. This manifests in a depression in Nusselt numbers. Therefore it is evident that the absolute value of Nusselt number for the non-slip case $(L=0)$ is markedly higher than the values for significant thermal slip $(L>0)$. The evolution in Nusselt number with a modification in Brinkman number, $\mathrm{Br}$, is shown in Fig. 5(c). It is apparent that Nusselt number magnitudes are enhanced with increasing Brinkman number. Magnitudes are considerably suppressed for the nondissipative case $(\mathrm{Br}=0)$. This phenomenon symbolizes the fact that the temperature gradient at the wall can be controlled by suitably manipulating Brinkman number (product of Prandtl and 
Eckert number). Since viscous heating is a realistic feature of electro-osmotic and magnetohydrodynamic convection flows, it is important to include this effect. Neglection of viscous heating (zero Brinkman number) clearly over-predicts wall heat transfer rates (Nusselt numbers). Figs.5(d-e) display the effect of both the Hartman number and thermal radiation parameter on the Nusselt number. There is a comparatively weak reduction in the Nusselt number occurs with greater Hartmann number $\left(M=\sqrt{\frac{\sigma \sigma}{\mu}} d_{2} B_{0}\right)$ and this is attributable to the increase in temperatures associated with stronger magnetic field. Greater thermal energy imparted to the body of the ionic nanoliquid results in an increase in heat transfer from the walls to the nanoliquid and a decrease in heat transfer to the walls. This reduces the Nusselt number at the micro-channel boundaries. Similarly, with increasing radiative parameter, $R n=\frac{16 \widetilde{\sigma} T_{0}^{3}}{3 \tilde{k} \mu c_{f}}$, there is also a depression in Nusselt numbers. Radiative heat flux energizes the electro-osmotic flow. It elevates temperatures since supplementary heat is added to the nanoliquid. This decreases the rate of heat transfer to the boundaries and manifests in a reduction in Nusselt number. The absolute value of Nusselt number is maximized in the absence thermal radiation (and also Hartmann magnetic number). Even a slight modification in radiative parameter exerts a marked influence on heat transfer characteristics throughout the length of the micro-channel and constitutes a useful mechanism for thermo-biological manipulation e.g. retinal irradiation with pulsed heating ${ }^{[34]}$. Overall, both magnetic field and thermal radiation therefore impart significant modifications to the convective heat transfer between the ionic nanoliquid and micro-channel boundaries. Fig. 5(f) illustrates the effects of Helmholtz-Smoluchowski velocity (Uhs) on the Nusselt number distribution. As $x$ increases from 0 to 2 with aligned axial electrical field $(U h s<0)$, the absolute value of Nusselt number is enhanced whereas with reverse axial electrical field $(U h s>0)$, the absolute value of Nusselt number is depleted. Fig. 5(g) shows that generally the Nusselt number is elevated for the case of an imposed heat source whereas it is reduced with a heat sink. The microchannel wall heat transfer characteristics are therefore highly sensitive to the presence of heat generation or heat absorption. 


\section{CONCLUDING REMARKS}

A theoretical study has been presented for peristaltic radiative-convective dissipative magnetohydrodynamic electro-osmotic propulsive flow of a viscous nano-liquid in an asymmetric microchannel under the dual action of an axial electrical field and transverse magnetic field. Convective boundary conditions, heat source and sink effects have been included. Brownian motion and thermophoresis are featured in the nanoscale model. The Rosseland diffusion flux approximation has been deployed to model thermal radiative heat flux. The conservation equations and boundary conditions are simplified by neglecting inertial forces and invoking the DebyeHückel linearization. The resulting governing equations are reduced into a system of nondimensional simultaneous ordinary differential equations, which is solved analytically. Numerical evaluation is performed with symbolic software (MATLAB). The influence of selected parameters on velocity, temperature, nano-particle concentration and Nusselt number are presented graphically. The present computations have shown that:

(i) With increasing thermal slip, the nano-particle volume fraction is increased whereas Nusselt number is reduced.

(ii) With increasing radiation parameter, the Nusselt number is generally reduced at the extremities of the micro-channel and the bulk flow is energized i.e. heated.

(iii) With increasing Brinkman (viscous heating) number, Nusselt numbers are increased.

(iv) For positive Helmholtz-Smoluchowski velocity (reverse axial electrical field) temperature is strongly decreased whereas for negative Helmholtz-Smoluchowski velocity (aligned axial electrical field) it is significantly elevated.

(v) Temperature profile decreases with higher values of slip parameter whereas the converse response is induced in nano-particle volume fraction profile for both heat source and heat sink parameter.

(vi) Heat source (generation) elevates temperatures whereas heat sink (absorption) depresses them, across the micro-channel span.

(vii) Heat sink elevates nano-particle volume fraction whereas heat source decreases it.

(viii) Increasing Hartmann (magnetic body force) parameter and Prandtl number enhance the nano-particle volume fraction.

(ix) Electro-osmotic body force strongly modifies the peristaltic transport phenomena.

(x) Increasing lower wall thermal Biot number generally enhances Nusselt numbers. 
(xi) Increasing Prandtl number generally enhances nano-particle volume fractions.

The present results provide a good foundation for further investigation into bio-inspired electromagnetohydrodynamic (EMHD) nanofluid pumping systems. The analysis has been confined to Newtonian viscous ionic nanoliquids. Future studies will consider non-Newtonian nanofluids.

\section{REFERENCES}

[1] Latham, T.W. (1996) Fluid motion in a peristaltic pump. MSc Thesis, Mechanical Engineering, MIT, Cambridge, USA.

[2] Shapiro, S.H., Jaffrin, M.Y. and S.L.Weinberg (1969) Peristaltic pumping with long wave lengths at low Reynolds number. J Fluid Mech, 37: p. 799-825.

[3] Fung, Y.C. and C.S. Yih (1968) Peristaltic transport. J Appl Mech, Trans ASME, 5: p. 669-675.

[4] Haroun, M.H. (2007) Non-linear peristaltic flow of a fourth-grade fluid in an inclined asymmetric channel. Comput Mater Sci, 39: p. 324-333.

[5] Mekheimer, Kh.S and Y.A. Elmaboud (2008) Peristaltic flow of a couple stress fluid in an annulus: application of an endoscope. Physica A, 387: p. 2403-2415.

[6] Hayat, T., Qureshi, M.U and N. Ali (2008) The influence of slip on the peristaltic motion of a third order fluid in an asymmetric channel. Phys Lett A, 372: p. 2653-2664.

[7] Anwar Bég, O., et al. (2013) Multi-Step DTM simulation of magneto-peristaltic flow of a conducting Williamson viscoelastic fluid, Int J Applied Mathematics Mechanics, 9 (12): p. 22-40.

[8] Srinivas, S and M. Kothandapani (2008) Peristaltic transport in an asymmetric channel with heat transfer - a note. Int Commun Heat Mass Transf, 35: p. 514-522.

[9] Kothandapani, M and S. Srinivas (2008) Nonlinear peristaltic transport of a Newtonian fluid in an inclined asymmetric channel through a porous medium. Phys Lett A, 372: p. 1265-1276.

[10] Hayat, T and N. Ali (2008) Effects of an endoscope on the peristaltic flow of a micropolar fluid. Math Comput Model, 48: p. 721-733.

[11] Vajravelu, K., Sreenadh,S., Hemadri, R and K. Murugasan (2009) Peristaltic transport of a Casson fluid in contact with a Newtonian fluid in a circular tube with permeable wall. Int J Fluid Mech Res, 36: p. $12-7$.

[12] Ali, N., Javid, K., Sajid, M., and O. Anwar Bég (2016) Numerical simulation of peristaltic flow of a biorheological fluid with shear-dependent viscosity in a curved channel. Computer Methods in Biomechanics and Biomedical Engineering, 19: p. 614-627. 
[13] Choi, S.U.S (1995) Enhancing thermal conductivity of fluid with nanoparticles developments and applications of non-Newtonian flow. ASME Fluids Engineering Division, 66: p. 99-105.

[14] Masuda, H., Ebata, A., Teramae, K and N. Hishinuma (1993) Alteration of thermal conductivity and viscosity of liquid by dispersing ultra-fine particles. Netsu Bussei, 7: p. 227-233.

[15] Eijkle, J.C.T and A.V.D. Berg (2005) Nanofluidics: what is it and what can we expect from it?. Microfluid Nanofluid, 1: p. 249-267.

[16] Ce, Y., Yang-Long, H and G. Song (2014) Nanomagnetism: Principles, nanostructures, and biomedical applications. Chinese Phys B, 23: p. 057505. doi:10.1088/1674-1056/23/5/057505.

[17] Xiu-Li, Y., Fang, M., and D. Zhi-Fei (2014) Multifunctional magnetic nanoparticles for magnetic resonance image-guided photothermal therapy for cancer. Chinese Phys B, 23: p. 044301. doi:10.1088/1674-1056/23/4/044301

[18] Buongiorno, J and W. Hu (2005) Nanofluid coolants for advanced nuclear power plants. Paper no. 5705, in: Proceedings of ICAPP '05, Seoul, May 15-19.

[19] Khan, W.A and I. Pop (2010) Boundary-layer flow of a nanofluid past a stretching sheet. Int J Heat Mass Transfer, 53: p. 2477-2483.

[20] Rana, P and R. Bhargava (2012) Flow and heat transfer of a nanofluid over a nonlinearly stretching sheet: a numerical study. Commun Nonlinear Sci Numer Simul, 17: p. 212-226.

[21] Mustafa, M., Hayat, T., Pop, I., Asghar, S and S. Obaidat (2011) Stagnation-point flow of a nanofluid towards a stretching sheet. Int J Heat Mass Transfer, 54: p. 5588-5594.

[22] Uddin, M.J., Kabir, M.N., Anwar Bég, O and Y. Alginahi (2018) Chebyshev collocation computation of magneto-bioconvection nanofluid flow over a wedge with multiple slips and magnetic induction. Proc. IMechE: Part N-Journal of Nanomaterials, Nanoengineering and Nanosystems, 232(4): p.109-122. doi: $10.1177 / 2397791418809795$

[23] Shamshuddin, M., Mishra, S.R., Ali Kadir and O. Anwar Bég (2019) Unsteady chemo-tribological squeezing flow of magnetized bioconvection lubricants: numerical study. J Nanofluids, 8 (2): p. 407-419. [24] Zohra, F.T., Uddin, M.J., Ismail, A.I., Anwar Bég, O and A. Kadir (2018) Boundary layer anisotropic slip magneto-bioconvection flow from a rotating cone to a nanofluid with Stefan blowing effects. Chinese J Physics, 56: p. 432-448.

[25] Anwar Bég, O., Sohail, A., Bég, T.A and A. Kadir (2018) B-spline collocation simulation of nonlinear transient magnetic nano-bio-tribological squeeze film flow. J Mechanics in Medicine and Biology, 18: p.1850007.1- 1850007.20.

[26] Zohra, F.T., Uddin, M.J., Ismail, A.I and O. Anwar Bég (2018) Bioconvective electromagnetic nanofluid transport from a wedge geometry: simulation of smart electro-conductive bio-nano-polymer processing. Heat Transfer-Asian Res, 47: p. 231-250. 
[27] Shukla, N., Rana, P., Anwar Bég, O., Kadir, A and Bani Singh (2018) Unsteady electromagnetic radiative nanofluid stagnation-point flow from a stretching sheet with chemically reactive nanoparticles, Stefan blowing effect and entropy generation. Proc. IMechE: Part N-Journal of Nanomaterials, Nanoengineering and Nanosystems, 232 (2-3): p. 69-82. doi: 10.1177/2397791418782030

[28] Tripathi, D., Akbar, N.S and O. Anwar Bég (2018) Transient peristaltic diffusion of nanofluids: A model for micropumps in medical engineering. J Hydrodynamics, 30(6): p. 774-781.

[29] Abbasi, F.M., et al. (2019) Entropy generation analysis for peristalsis of nanofluid with temperature dependent viscosity and Hall effects. J Magnetism and Magnetic Materials, 474: p.434-441.

[30] Akbar, N.S., Tripathi, D and O. Anwar Bég (2015) Modelling nanoparticle geometry effects on peristaltic pumping of medical magnetohydrodynamic nanofluids with heat transfer. $\mathrm{J}$ Mechanics in Medicine and Biology, 16 (2): p.1650088.1-1650088.20.

[31] Hottel, H.C., and A. F. Sarofim (1967) Radiative Transfer. McGraw-Hill Book Company, New York, USA.

[32] Larry Bodgi, et al., (2016) Mathematical models of radiation action on living cells: From the target theory to the modern approaches. A historical and critical review. J Theoretical Biology, 394: p. 93-101.

[33] Eisenberg, D.P., Bischof, J.C and Y. Rabin (2015) Thermomechanical stress in cryopreservation via vitrification with nanoparticle heating as a stress-moderating effect. ASME J Biomech Eng., 138(1): p. 011010-011010-8.

[34] Ennemoser, A and N. Hausmann (2008) Regulation of human eye growth by the energy density of the radiation in the retina-A biophysical theory in ophthalmology. J Theoretical Biology, 253: p. 302-2309.

[35] Sekimura, T (1995) The diversity in shoot morphology of herbaceous plants in relation to solar radiation captured by leaves. J Theoretical Biology, 177: p. 289-298.

[36] Said, Z., et al. (2013) Radiative properties of nanofluids. International Communications in Heat and Mass Transfer, 46: p. 74-84.

[37] Zhang, L., et al. (2014) Radiative properties of ionic liquid-based nanofluids for medium-to-hightemperature direct absorption solar collectors. Solar Energy Materials and Solar Cells, 130: p. 521-528.

[38] Prakash, J., Siva, E.P., Tripathi, D., Kuharat, S and O. Anwar Bég (2018) Peristaltic pumping of magnetic nanofluids with thermal radiation and temperature-dependent viscosity effects: modelling a solar magneto-biomimetic nanopump. Renewable Energy, 133: p. 1308-1326. doi.org/10.1016/j.renene.2018.08.096 0960-1481

[39] Mehmood, R., Tabassum, R., Kuharat, S., Anwar Bég, O and M. Babaie (2018) Thermal slip in oblique radiative nano-polymer gel transport with temperature-dependent viscosity: solar collector nanomaterial coating manufacturing simulation. Arabian J Science and Engineering, 44(2): p. 1525-1541. doi:10.1007/s13369-018-3599-y 
[40] Kuharat, S and O. Anwar Bég (2018) Simulation of a nanofluid-based annular solar collector, ICHTFM 2018: 20th International Conference on Heat Transfer and Fluid Mechanics. Istanbul, Turkey, August 16 17.

[41] Hasona, W.M., Almalki, N.H., ElShekhipy A.A., and M.G. Ibrahim, Thermal radiation and variable electrical conductivity effects on MHD peristaltic motion of Carreau nanofluids: Radiotherapy and thermotherapy of oncology treatment, Heat transfer Asian Research, 48 (3) 2019, 938-956.

[42] Sudarsana Reddy, P., Sreedevi, P., and A.J. Chamkha, Heat and mass transfer flow of a nanofluid over an inclined plate under enhanced boundary conditions with magnetic field and thermal radiation, Heat transfer Asian research, 46 (7) 2017, 815-839.

[43] Sudarsana Reddy, P., A. J. Chamkha, A.J., and A. Al-Mudhaf, MHD heat and mass transfer flow of a nanofluid over an inclined vertical porous plate with radiation and heat generation/absorption, Advanced Powder Technology, 28 (2017) 1008-1017.

[44] Barz, D.P.J and P. Ehrhard (2005) Model and verification of electrokinetic flow and transport in a micro-electrophoresis device, Lab Chip, 5: p. 949 - 958.

[45] Chang, J.S and A. Watson (1994) Electromagnetic hydrodynamics. IEEE Trans. Dielectr. Electr Insul, 1 (5): p. 871-895.

[46] Bandopadhyay, A., Shaik, V.A and S. Chakraborty (2015) Effects of finite ionic size and solvent polarization on the dynamics of electrolytes probed through harmonic disturbance. Phys Rev E, 91: p. 042307.

[47] Tripathi, D., Bhushan, S and O. Anwar Bég (2017) Analytical study of electroosmosis modulated capillary peristaltic hemodynamics. Journal of Mechanics in Medicine and Biology, 17(3): p. 1750052.

[48] Tripathi, D., Bhushan, S and O. Anwar Bég (2018) Unsteady viscous flow driven by the combined effects of peristalsis and electro-osmosis. Alexandria Engineering Journal, 57(3): p. 1349-1359.

[49] Schit, G.C., Mondal, A., Sinha, A and P.K. Kundu (2016) Effects of slip velocity on rotating electroosmotic flow in a slowly varying micro-channel. Colloids Surf A, 489: p. 249-255.

[50] Guo, X and H. Qi (2017) Analytical solution of electro-osmotic peristalsis of fractional Jeffreys fluid in a micro-channel. Micromachines, 8(12): p. 341.

[51] Schit, G.C., Mondal, A., Sinha, A and P.K. Kundu (2016) Electro-osmotically driven MHD flow and heat transfer in micro-channel. Physica A, 449: p. 437-454.

[52] Prakash, J., Sharma, A and D. Tripathi (2018) Thermal radiation effects on electroosmosis modulated peristaltic transport of ionic nanoliquids in biomicrofluidics channel. Journal of Molecular Liquids, 249: p. 843-855. 
[53] Tripathi, D., Sharma, A and O. Anwar Bég (2018) Joule heating and buoyancy effects in electroosmotic peristaltic transport of aqueous nanofluids through a micro-channel with complex wave propagation, Advanced Powder Technology, 29: p. 639-653.

[54] Prakash, J., Ramesh, K., Tripathi, D and R. Kumar (2018) Numerical simulation of heat transfer in blood flow altered by electroosmosis through tapered micro-vessels. Microvascular Research, 118: p. 162172.

[55] Alizadeh, A., et al. (2014) Mixing enhancement of low-Reynolds electro-osmotic flows in microchannels with temperature-patterned walls. J. Colloid and Interface Science, 431: p. 50-63.

[56] Chien-Chih Huang, Martin Z. Bazant and Todd Thorsen (2010) Ultrafast high-pressure AC electroosmotic pumps for portable biomedical microfluidics. Lab Chip, 1: p. 80-85.

[57] Vafai, R.H., et al., (2013) An electro-osmotically driven micromixer modified for high miniaturized microchannels using surface micromachining. Biotechnology and Bioprocess Engineering, 18(3): p. 594605.

[58] Arruebo, M., et al. (2007) Magnetic nanoparticles for drug delivery. Nanotoday, 2(3): p. 22-32.

[59] Funk RH, Monsees T, Ozkuour N (2009). Electromagnetic effects-from cell biology to medicine. Prog Histochem Cytochem. 43(4):177-264.

[60] J.M. Min et al. (2004) On the efficiency of electrokinetic pumping of liquids through nanoscale channels, Sensors and Actuators B: Chemical, 98, 368-277.

[61] T. Bourouina and J.P. Grandchamp (1996). Modeling micropumps with electrical equivalent networks, J. Micromech. Microeng., 6, 398-404.

[62] M. Busek, M. Nötzel, C. Polk, and F. Sonntag (2013), Characterization and simulation of peristaltic micropumps J. Sens. Sens. Syst., 2, 165-169.

[63] Lightfoot, E. N. (1974). Transport Phenomena and Living Systems: Biomedical Aspects of Momentum and Mass Transport (1 ed.). John Wiley \& Sons, New York, USA.

[64] E. Moros (Ed.), (2012) Physics of Thermal Therapy: Fundamentals and Clinical Applications, CRC Press, Florida, USA.

\section{ACKNOWLEDGEMENTS}

The authors acknowledge the comments of the reviewers which have improved the clarity of the present work. 


\section{APPENDIX:}

$$
\begin{aligned}
& A=-\frac{e^{-\kappa h_{1}}}{e^{\kappa\left(h_{1}-h_{2}\right)}-e^{-\kappa\left(h_{1}-h_{2}\right)}}, B=-\frac{e^{-\kappa h_{1}}}{e^{\kappa\left(h_{1}-h_{2}\right)}-e^{-\kappa\left(h_{1}-h_{2}\right)}}, f_{1}=\frac{N t+B h_{1} B h_{2} N b}{N t\left(B h_{1}+B h_{2}+B h_{1} B h_{2}\left(h_{2}-h_{1}\right)\right)}, \\
& N_{1}=\kappa^{2}-M^{2}, N_{2}=\frac{U h s B}{N_{1}}, N_{3}=-\frac{U h s . A}{N_{1}}, N_{4}=\left(M-M^{2} L\right) e^{M h_{1}}, N_{5}=-\left(M+M^{2} L\right) e^{-M h_{1}}, \\
& N_{6}=N_{2} \kappa(1+\kappa L) e^{-\kappa h_{1}}-N_{3} \kappa(1-\kappa L) e^{-\kappa h_{1}}-1, N_{7}=\left(M+M^{2} L\right) e^{M h_{2}}, N_{8}=\left(M^{2} L-M\right) e^{M h_{2}}, \\
& N_{9}=N_{2} \kappa(1-\kappa L) e^{-\kappa h_{2}}-N_{3} \kappa(1+\kappa L) e^{-\kappa h_{2}}-1, N_{10}=-\frac{F}{2}-N_{2} e^{-\kappa h_{1}}-N_{3} e^{\kappa h_{1}}, \\
& N_{11}=\frac{F}{2}-N_{2} e^{-\kappa h_{2}}-N_{3} e^{\kappa h_{2}}, N_{12}=N_{4}\left(h_{2}-h_{1}\right)-\left(e^{-M h_{2}}-e^{-M h_{1}}\right), N_{13}=N_{5}\left(h_{2}-h_{1}\right)-\left(e^{-M h_{2}}-e^{-M h_{1}}\right) \\
& \text {, } N_{14}=N_{6}\left(h_{2}-h_{1}\right)-\left(N_{11}-N_{10}\right), N_{15}=N_{7}\left(h_{2}-h_{1}\right)-\left(e^{M h_{2}}-e^{M h_{1}}\right), N_{16}=N_{8}\left(h_{2}-h_{1}\right)-\left(e^{-M h_{2}}-e^{-M h_{1}}\right) \text {, } \\
& N_{17}=N_{9}\left(h_{2}-h_{1}\right)-\left(N_{11}-N_{10}\right), N_{18}=\frac{N_{16} N_{12}-N_{13} N_{15}}{N_{15}}, N_{19}=\frac{N_{17} N_{12}-N_{14} N_{15}}{N_{15}}, N_{21}=\frac{N b \cdot \operatorname{Pr} \cdot f_{1}}{1+R n \cdot \operatorname{Pr}}, \\
& N_{22}=-\left(\frac{B r}{1+R n \cdot \operatorname{Pr}}\right), N_{23}=-\left(\frac{\beta \cdot \operatorname{Pr}}{1+R n \cdot \operatorname{Pr}}\right), N_{24}=\frac{N_{22} M^{4} c_{3}^{2}}{4 M^{2}+2 M N_{21}}, N_{25}=\frac{N_{22} M^{4} c_{4}^{2}}{4 M^{2}-2 M N_{21}}, \\
& N_{26}=\frac{N_{22} N_{2}^{2} \kappa^{4}}{4 \kappa^{2}-2 \kappa N_{21}}, N_{27}=\frac{N_{22} N_{3}^{2} \kappa^{4}}{4 \kappa^{2}+2 \kappa N_{21}}, N_{28}=\frac{N_{22}\left(2 c_{3} c_{4} M^{4}+2 N_{2} N_{3} \kappa^{4}\right)+N_{23}}{N_{21}}, \\
& N_{29}=\frac{2 N_{22} c_{3} M^{2} N_{2} \kappa^{2}}{(M-\kappa)^{2}+N_{21}(M-\kappa)}, N_{30}=\frac{2 N_{22} c_{3} M^{2} N_{3} \kappa^{2}}{(M+\kappa)^{2}+N_{21}(M+\kappa)}, N_{31}=\frac{2 N_{22} c_{4} M^{2} N_{3} \kappa^{2}}{\left(N_{1}+\kappa\right)^{2}-N_{21}(M+\kappa)}, \\
& N_{32}=\frac{2 N_{22} M^{2} N_{3} \kappa^{2}}{(\kappa-M)^{2}+N_{21}(\kappa-M)} \\
& N_{33}=2 N_{24} M e^{2 M h_{1}}-2 N_{25} M e^{-2 M h_{1}}-2 \kappa N_{26} e^{-2 \kappa h_{1}}+2 \kappa N_{27} e^{2 \kappa h_{1}}+N_{28}+(\kappa-M) N_{32} e^{(\kappa-M) h_{1}} \\
& +N_{29}(M-\kappa) e^{(M-\kappa) h_{1}}+N_{30}(M+\kappa) e^{(M+\kappa) h_{1}}-N_{31}(M+\kappa) e^{-(M+\kappa) h_{1}} \\
& -B h_{1}\left(\begin{array}{l}
N_{24} e^{2 M h_{1}}+N_{25} e^{-2 M h_{1}}+N_{26} e^{-2 \kappa h_{1}}+N_{27} e^{2 \kappa h_{1}}+N_{28} h_{1} \\
+N_{29} e^{(M-\kappa) h_{1}}+N_{30} e^{(M+\kappa) h_{1}}+N_{31} e^{-(M+\kappa) h_{1}}+N_{32} e^{(\kappa-M) h_{1}}
\end{array}\right) \\
& N_{34}=-N_{21} e^{-N_{21} h_{1}}-B h_{1} e^{-N_{21} h_{1}}, N_{35}=-N_{21} e^{-N_{21} h_{2}}+B h_{2} e^{-N_{21} h_{2}},
\end{aligned}
$$




$$
\begin{aligned}
& N_{36}=2 N_{24} M e^{2 M h_{2}}-2 N_{25} M e^{-2 M h_{2}}-2 \kappa N_{26} e^{-2 \kappa h_{2}}+2 \kappa N_{27} e^{2 \kappa h_{2}}+N_{28}+(\kappa-M) N_{32} e^{(\kappa-M) h_{2}} \\
& +N_{29}(M-\kappa) e^{(M-\kappa) h_{2}}-N_{30}(M+\kappa) e^{(M+\kappa) h_{1}}-N_{31}(M+\kappa) e^{-(M+\kappa) h_{2}} \\
& +B h_{2}\left(\begin{array}{l}
N_{24} e^{2 M h_{2}}+N_{25} e^{-2 M h_{2}}+N_{26} e^{-2 \kappa h_{2}}+N_{27} e^{2 \kappa h_{2}}+N_{28} h_{2} \\
+N_{29} e^{(M-\kappa) h_{2}}+N_{30} e^{(M+\kappa) h_{2}}+N_{31} e^{-(M+\kappa) h_{2}}+N_{32} e^{(\kappa-M) h_{2}}
\end{array}\right), \\
& c_{1}=N_{10}-h_{1} c_{2}-e^{M h_{1}} c_{3}-e^{-M h_{1}} c_{4}, c_{2}=\frac{1}{h_{2}-h_{1}}\left[N_{11}-N_{10}-\left(e^{M h_{2}}-e^{M h_{1}}\right) c_{3}-\left(e^{-M h_{2}}-e^{-M h_{1}}\right) c_{4}\right], \\
& c_{3}=\frac{N_{14}-N_{13} c_{4}}{N_{12}}, c_{4}=\frac{N_{19}}{N_{18}}, c_{5}=\frac{N_{33}+N_{34} c_{6}}{B h_{1}}, c_{6}=\frac{\left(1-N_{36}\right) B h_{1}-N_{33} B h_{2}}{B h_{2} N_{34}+B h_{1} N_{35}}, c_{7}=\frac{N_{36}-N_{38}}{h_{1}-h_{2}} \text {, } \\
& c_{8}=N_{37}-c_{7} h_{1}, N_{37}=\frac{N t}{N b}\left(\begin{array}{c}
c_{5}+c_{6} e^{N_{21} h_{1}}+N_{24} e^{2 M h_{1}}+N_{25} e^{-2 M h_{1}}+N_{26} e^{-2 \kappa h_{1}}+N_{27} e^{2 \kappa h_{1}}+N_{28} h_{1} \\
+N_{29} e^{(M-\kappa) h_{1}}+N_{30} e^{(M+\kappa) h_{1}}+N_{31} e^{-(M+\kappa) h_{1}}+N_{32} e^{(\kappa-M) h_{1}} \theta
\end{array}\right), \\
& N_{38}=1+\frac{N t}{N b}\left(\begin{array}{c}
c_{5}+c_{6} e^{N_{21} h_{2}}+N_{24} e^{2 M h_{2}}+N_{25} e^{-2 M h_{2}}+N_{26} e^{-2 \kappa h_{2}}+N_{27} e^{2 \kappa h_{2}}+N_{28} h_{2} \\
+N_{29} e^{(M-\kappa) h_{2}}+N_{30} e^{(M+\kappa) h_{2}}+N_{31} e^{-(M+\kappa) h_{2}}+N_{32} e^{(\kappa-M) h_{2}}
\end{array}\right)
\end{aligned}
$$




\section{FIGURES}

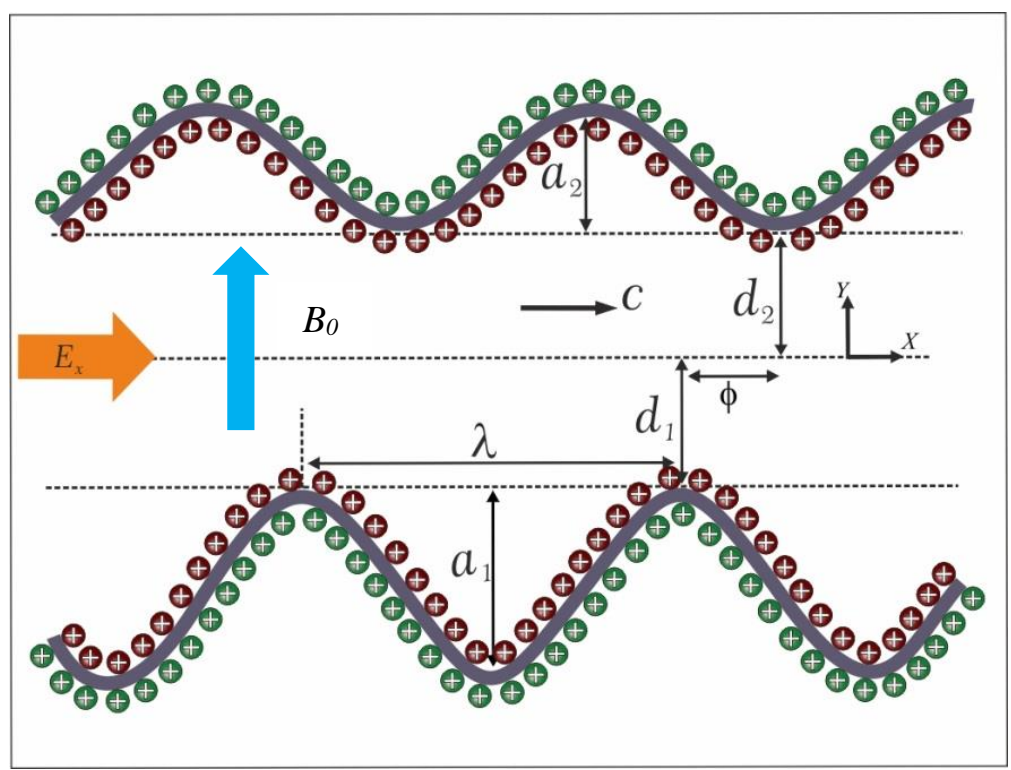

Fig.1.Geometry for nanoliquid electro-osmotic magnetohydrodynamic flow through an asymmetric microchannel

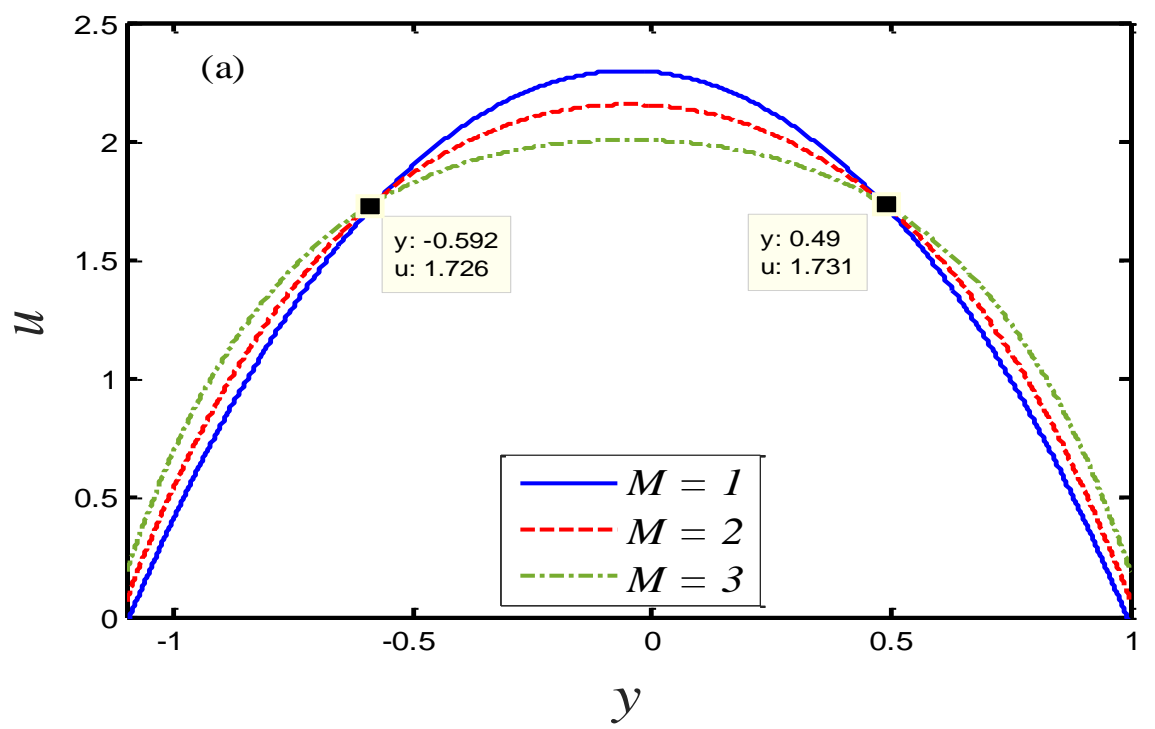

Fig.2.(a). Effect of Hartmann (magnetic field) number $(M)$ on axial velocity for $a=0.3, b=0.2, \phi=\pi / 4$, $x=0.5, d=1, \kappa=0.2, L=0.1, \Theta=1.5$, Uhs $=2$. 


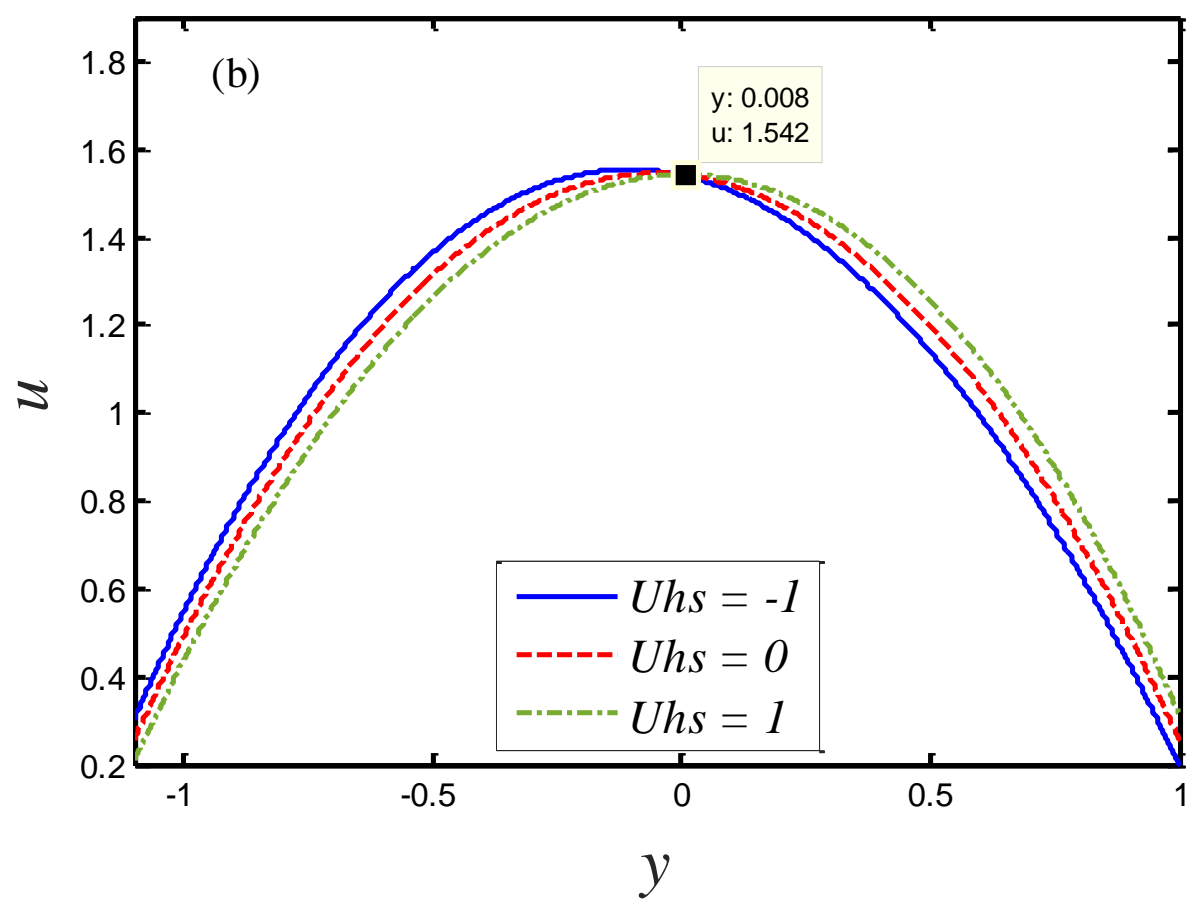

Fig.2.(b). Effect of Helmholtz-Smoluchowski velocity (Uhs) on axial velocity for $a=0.3, b=0.2$, $\phi=\pi / 4, x=0.5, d=1, \kappa=0.2, L=0.1, \Theta=1.5, M=2$.

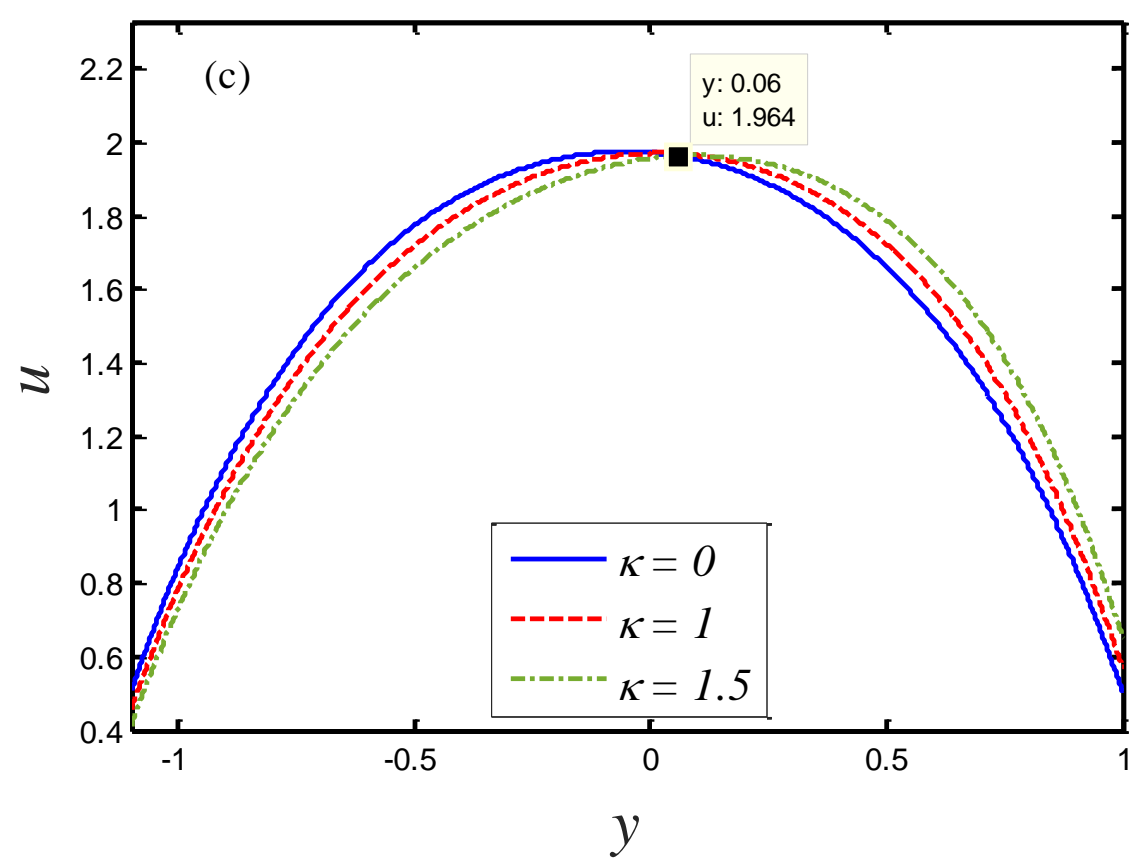

Fig.2. (c). Effect of Debye-Hückel parameter ( $\kappa$ ) on axial velocity for $a=0.3$, $b=0.2, \phi=\pi / 4, x=0.5, d=1, L=0.1, \Theta=1.5, M=2, U h s=2$. 


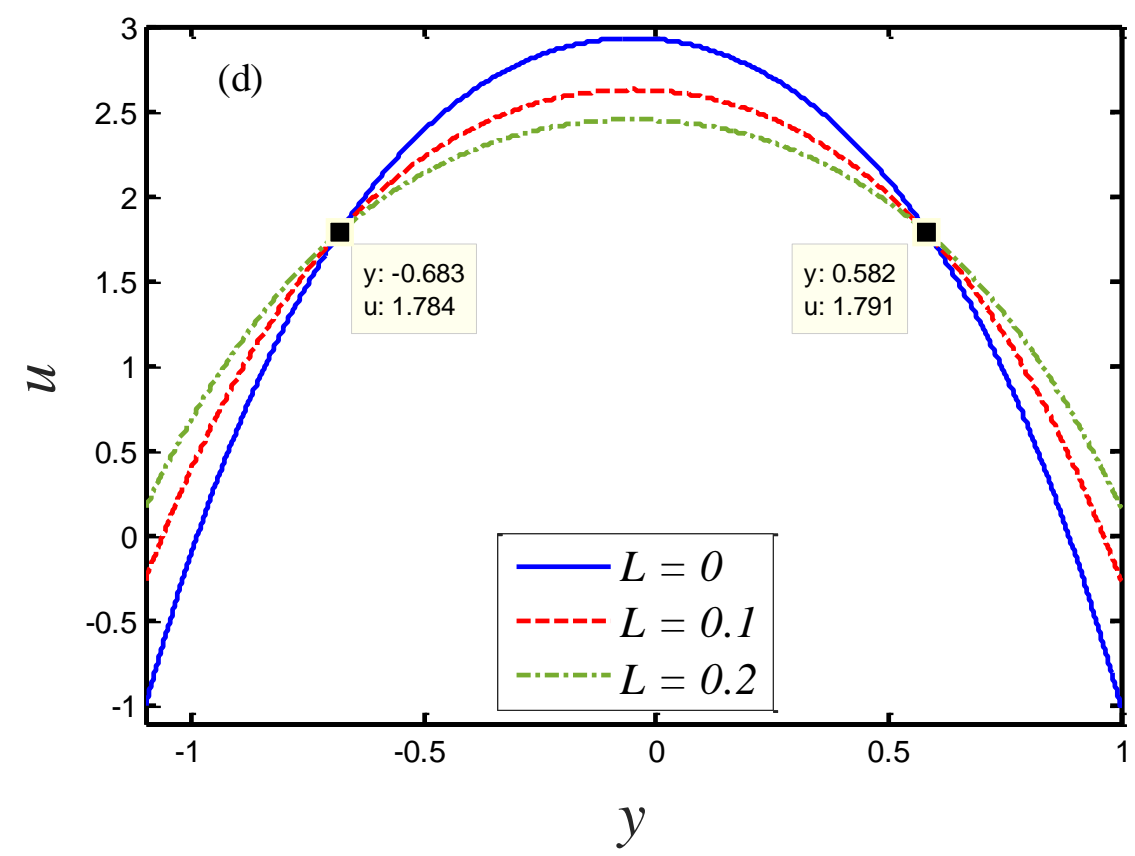

Fig.2.(d). Effect of thermal slip parameter $(L)$ on axial velocity for $a=0.3, b=0.2$, $\phi=\pi / 4, x=0.5, d=1, \Theta=1.5, M=2, \kappa=0.2$, Uhs $=2$.

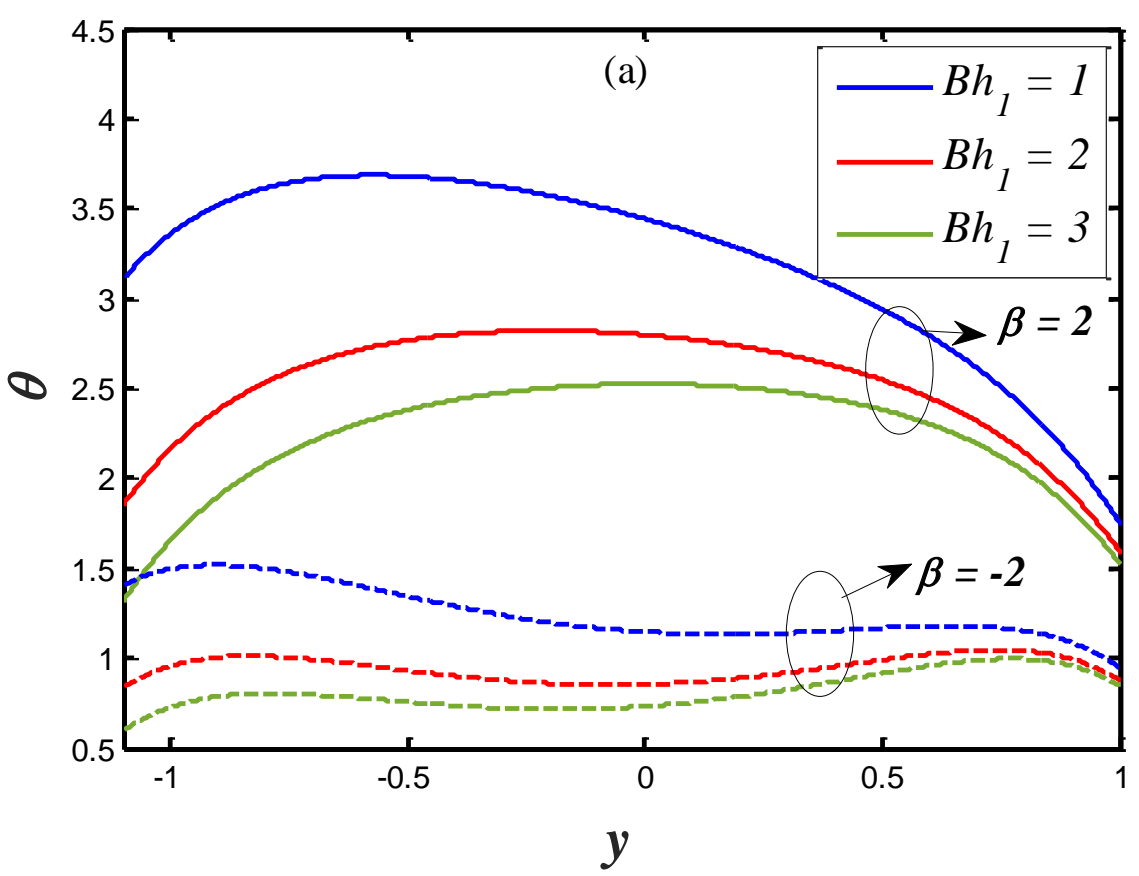

Fig.3.(a). Effect of lower wall thermal Biot number $\left(B h_{1}\right)$ and heat source/sink $(\beta)$ on temperature distribution for $a=0.3, b=0.2, \phi=\pi / 4, x=0.5, d=1, \Theta=1.5, M=2, \kappa=0.5, U h s=2, L=0.3, N t=0.6, N b=0.6, B h_{2}=3, \operatorname{Pr}=0.71$, $R n=1.5, B r=3$, Uhs $=2$. 


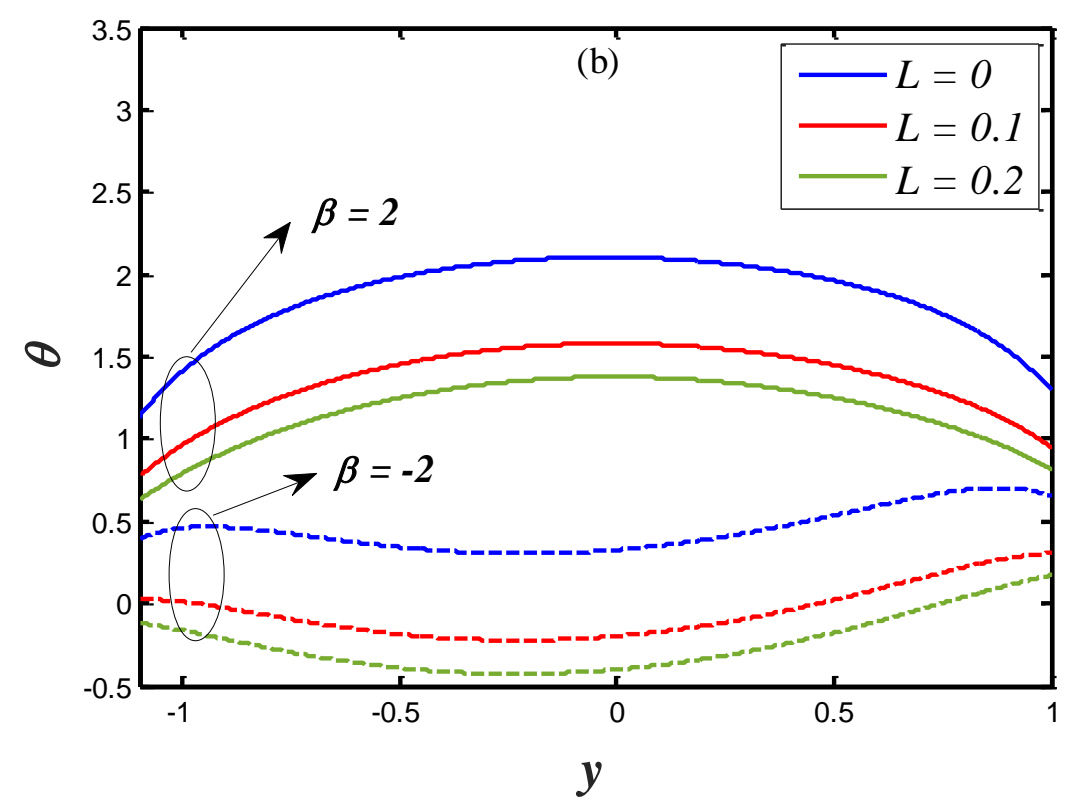

Fig.3.(b). Effect of thermal slip parameter $(L)$ and heat source/sink $(\beta)$ on temperature distribution for $a=0.3, b=0.2$, $\phi=\pi / 4, x=0.5, d=1, \Theta=1.5, M=2, \kappa=0.5$, Uhs $=2, L=0.3, N t=0.6, N b=0.6, B h_{1}=3, B h_{2}=3, \operatorname{Pr}=0.71, R n=$ $1.5, \mathrm{Br}=3, \mathrm{Uhs}=2$.

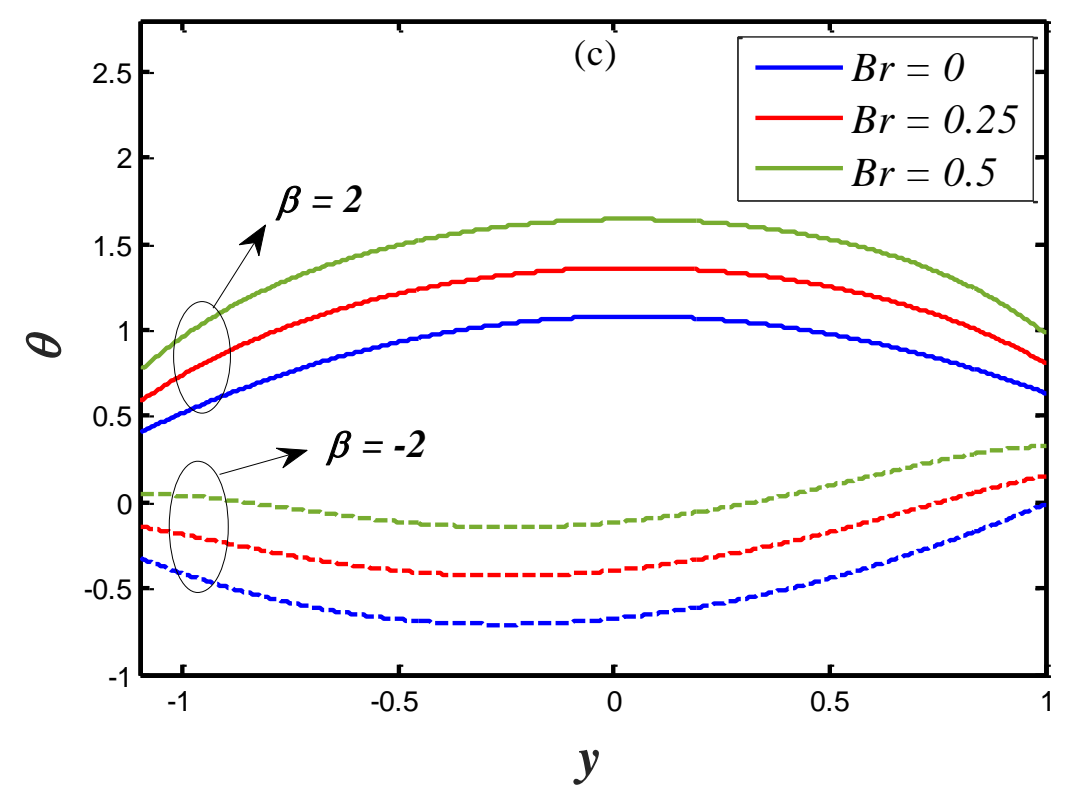

Fig.3.(c). Effect of Brinkman number $(B r)$ and heat source/sink $(\beta)$ on temperature distribution for $a=0.3, b=0.2$, $\phi=\pi / 4, x=0.5, d=1, \Theta=1.5, M=2, \kappa=0.5, U h s=2, L=0.3, N t=0.6, N b=0.6, B h_{1}=3, B h_{2}=3, \operatorname{Pr}=0.71, R n=$ $1.5, M=2$, Uhs $=2$. 


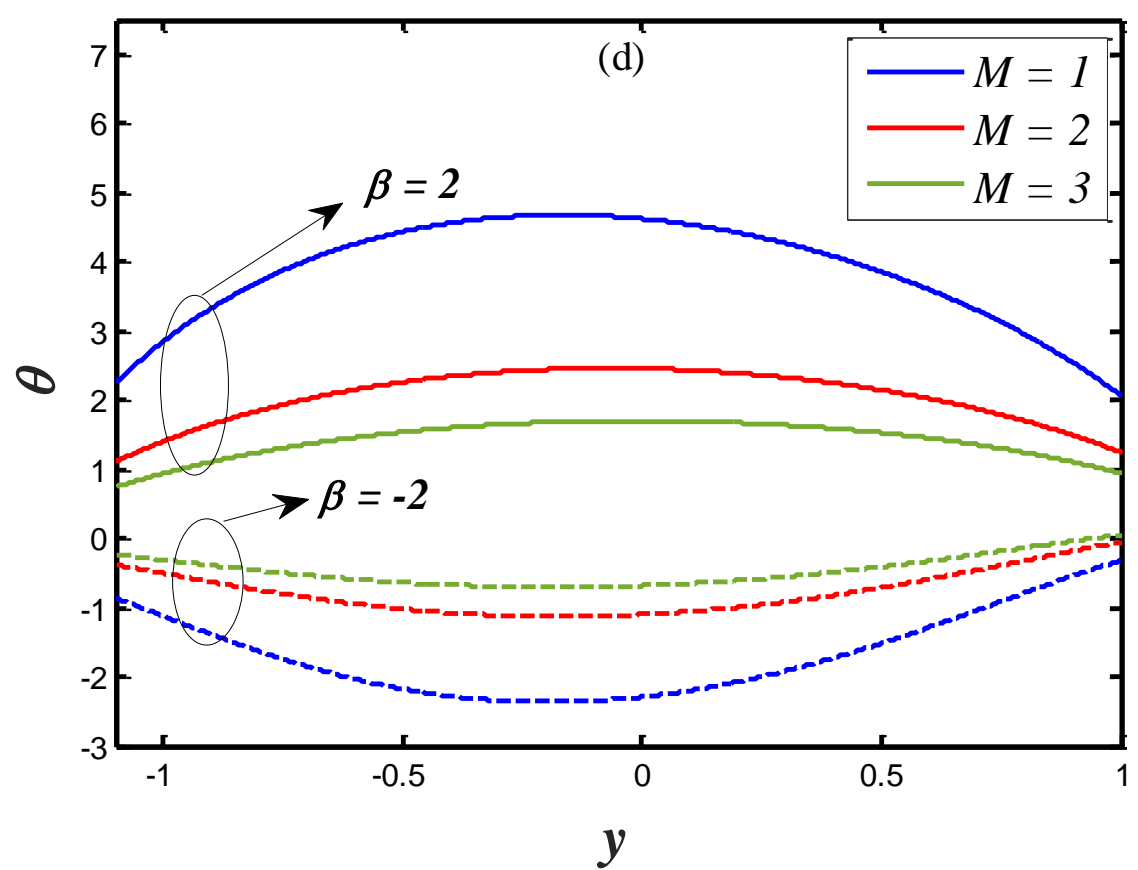

Fig.3.(d). Effect of Hartmann magnetic number $(M)$ and heat source/sink $(\beta)$ on temperature distribution for $a=0.3$, $b=0.2, \phi=\pi / 4, x=0.5, d=1, \Theta=1.5, \kappa=0.5, U h s=2, L=0.3, N t=0.6, N b=0.6, B h_{1}=3, B h_{2}=3, \operatorname{Pr}=0.71, R n=$ $1.5, \mathrm{Br}=3$, Uhs $=2$.

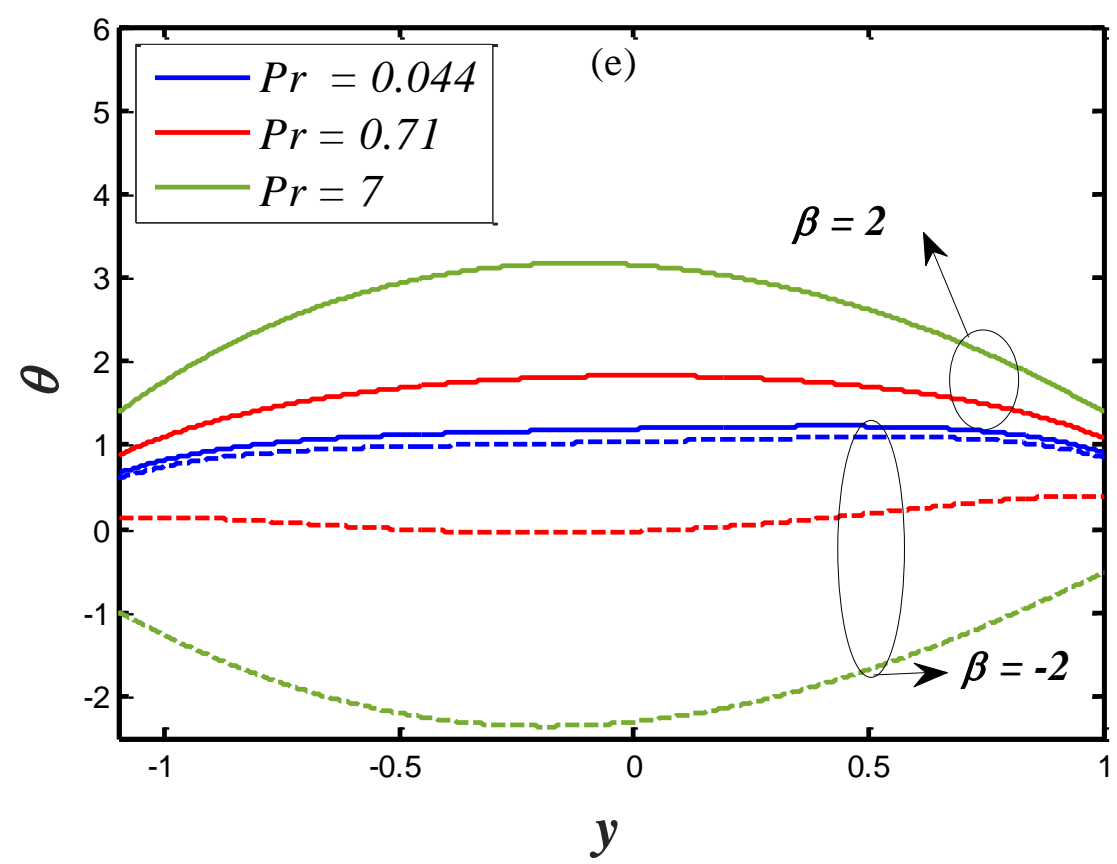

Fig.3.(e). Effect of Prandtl number $(\operatorname{Pr})$ and heat source/sink $(\beta)$ on temperature distribution for $a=0.3, b=0.2$, $\phi=\pi / 4, x=0.5, d=1, \Theta=1.5, M=2, \kappa=0.5, U h s=2, L=0.3, N t=0.6, N b=0.6, B h_{1}=3, B h_{2}=3, R n=1.5, B r=3$. 


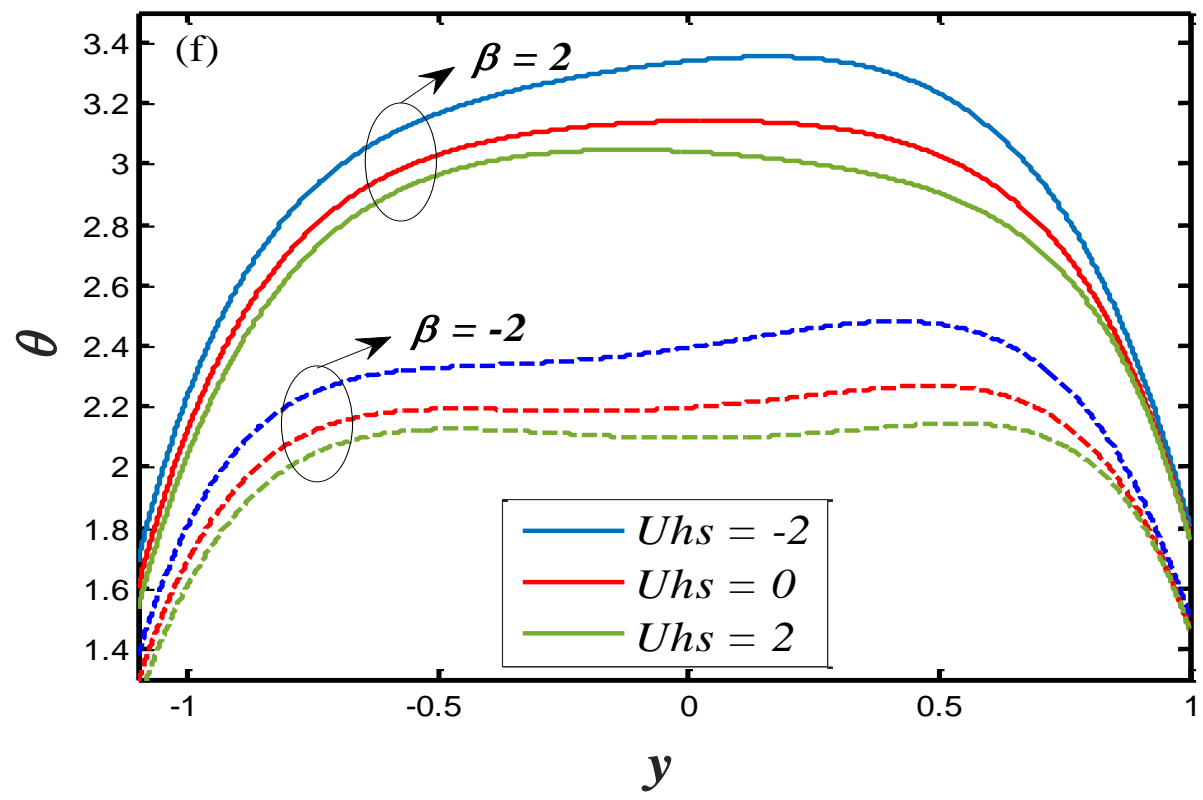

Fig.3.(f). Effect of Helmholtz-Smoluchowski velocity (Uhs) and heat source/sink $(\beta)$ on temperature distribution for $a=0.3, b=0.2, \phi=\pi / 4, x=0.5, d=1, \Theta=1.5, M=2, \kappa=0.5, L=0.3, N t=0.6, N b=0.6, B h_{1}=3, B h_{2}=3, \operatorname{Pr}=0.71$, $R n=1.5, B r=3$.

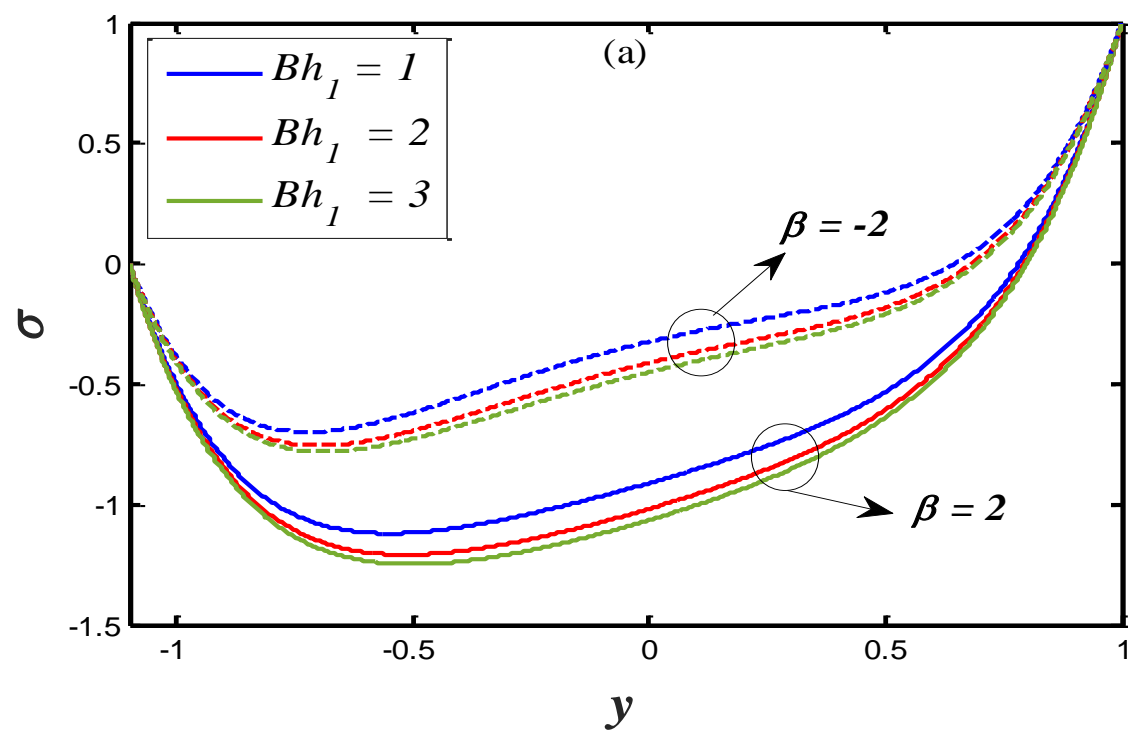

Fig.4.(a). Effect of lower wall thermal Biot number $\left(B h_{1}\right)$ and heat source/sink $(\beta)$ on nanoparticle volume fraction for $a=0.3, b=0.2, \phi=\pi / 4, x=0.5, d=1, \Theta=1.5, M=2, \kappa=0.2, U h s=2, L=0.3, N t=0.6, N b=0.9, B h_{2}=2, P r=$ $0.71, R n=1, B r=5$. 


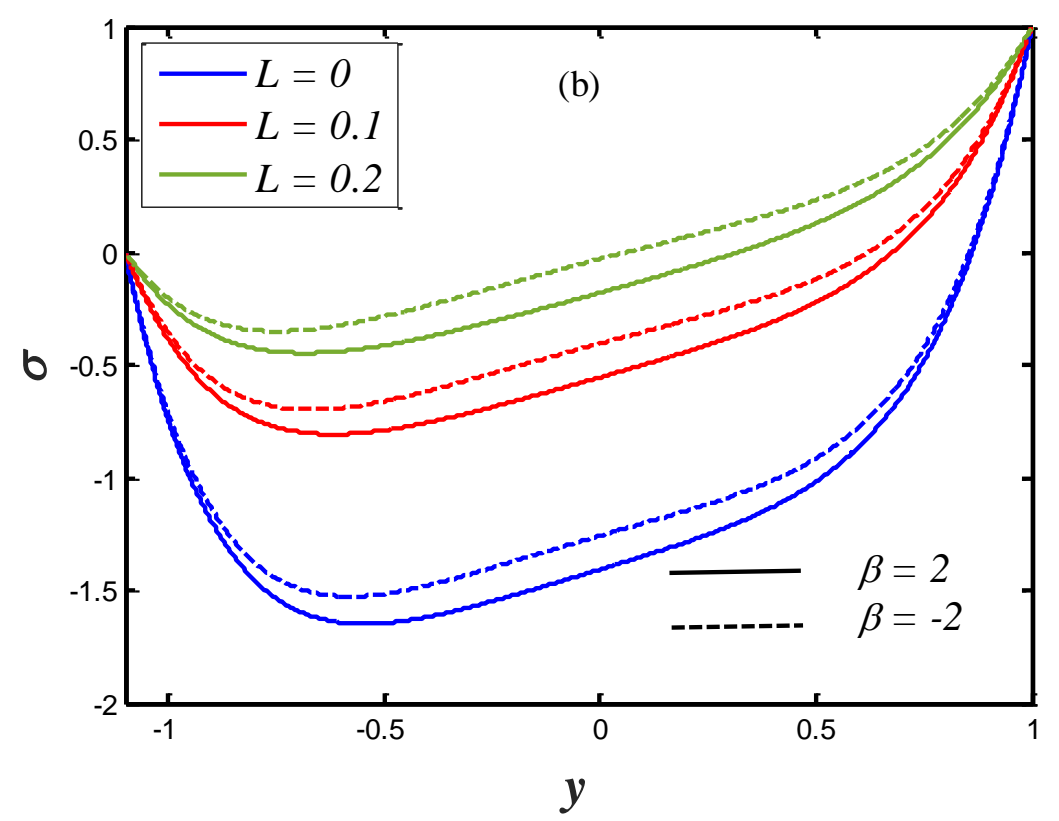

Fig.4.(b). Effect of thermal slip parameter $(L)$ and heat source/sink $(\beta)$ on nanoparticle volume fraction for $a=0.3$, $b=0.2, \phi=\pi / 4, x=0.5, d=1, \Theta=2, M=2, \kappa=0.2, U h s=2, N t=0.6, N b=1.6, B h_{2}=3, B h_{2}=3, P r=0.71, R n=4, B r$ $=7$.

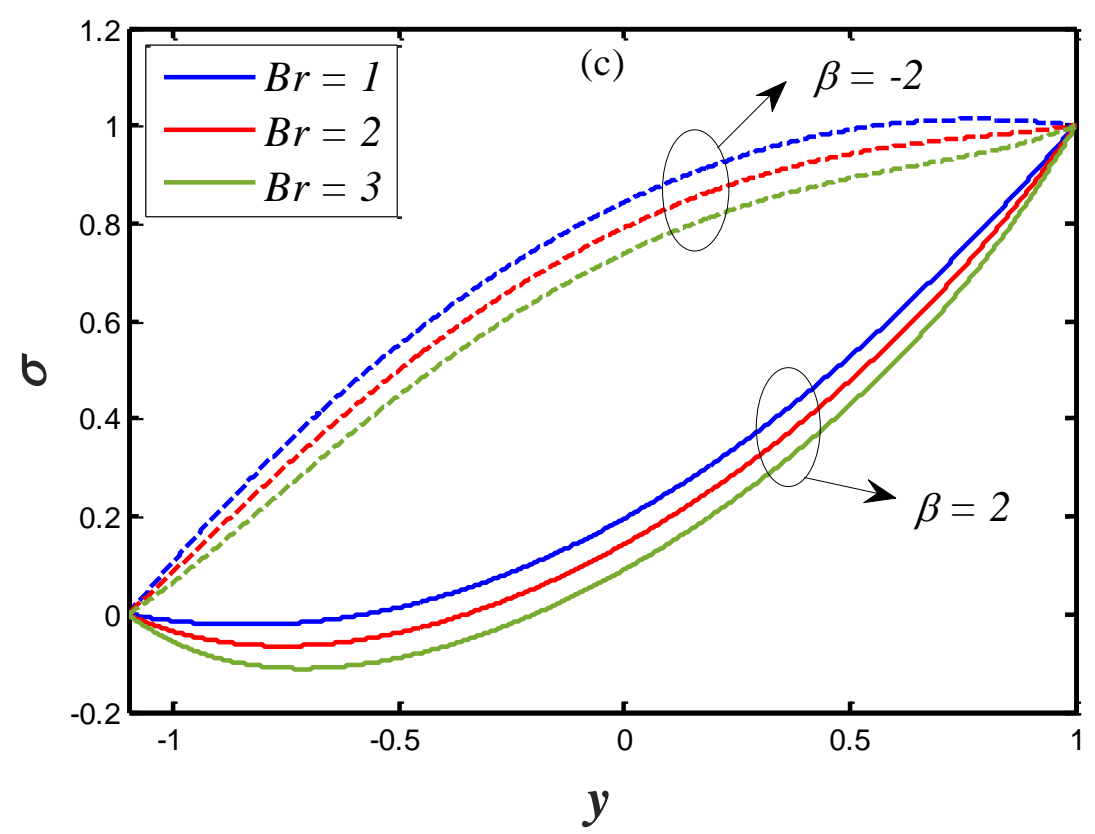

Fig.4.(c). Effect of Brinkman number $(B r)$ and heat source/sink $(\beta)$ on nanoparticle volume fraction for $a=0.3$, $b=0.2, \phi=\pi / 4, x=0.5, d=1, \Theta=2, M=2, \kappa=0.2, U h s=2, L=0.3, N t=0.6, N b=0.6, B h_{1}=3, B h_{2}=3, P r=0.71$, $R n=2$. 


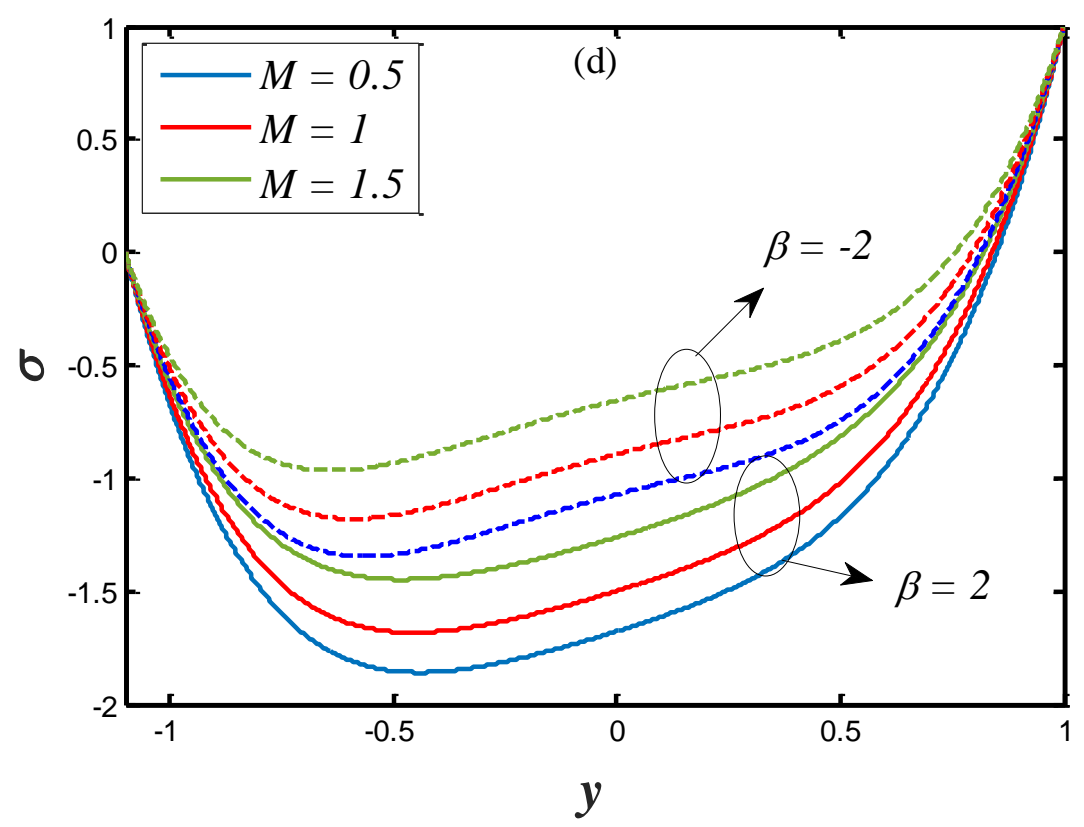

Fig.4.(d). Effect of Hartmann magnetic number $(M)$ and heat source/sink $(\beta)$ on nanoparticle volume fraction for $a=0.3, b=0.2, \phi=\pi / 4, x=0.5, d=1, \Theta=2, M=2, \kappa=0.2, U h s=2, L=0.3, N t=0.6, N b=0.6, B h_{1}=3, B h_{2}=3, \operatorname{Pr}=$ $0.71, R n=2$.

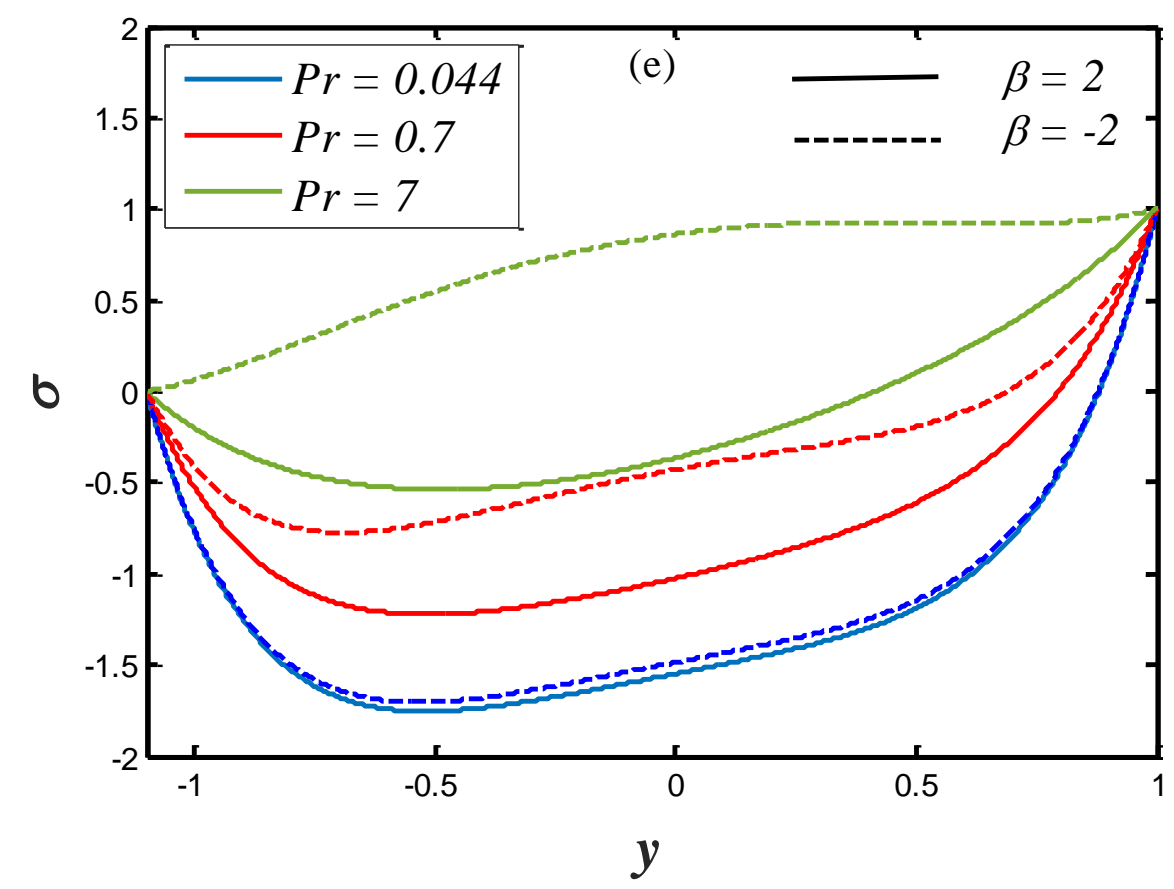

Fig.4.(e). Effect of Prandtl number $(\operatorname{Pr})$ and heat source/sink $(\beta)$ on nanoparticle volume fraction for $a=0.3, b=0.2$, $\phi=\pi / 4, x=0.5, d=1, \Theta=2, M=2, \kappa=0.2, U h s=2, L=0.3, N t=0.6, N b=0.6, B h_{1}=3, B h_{2}=3, R n=2$. 


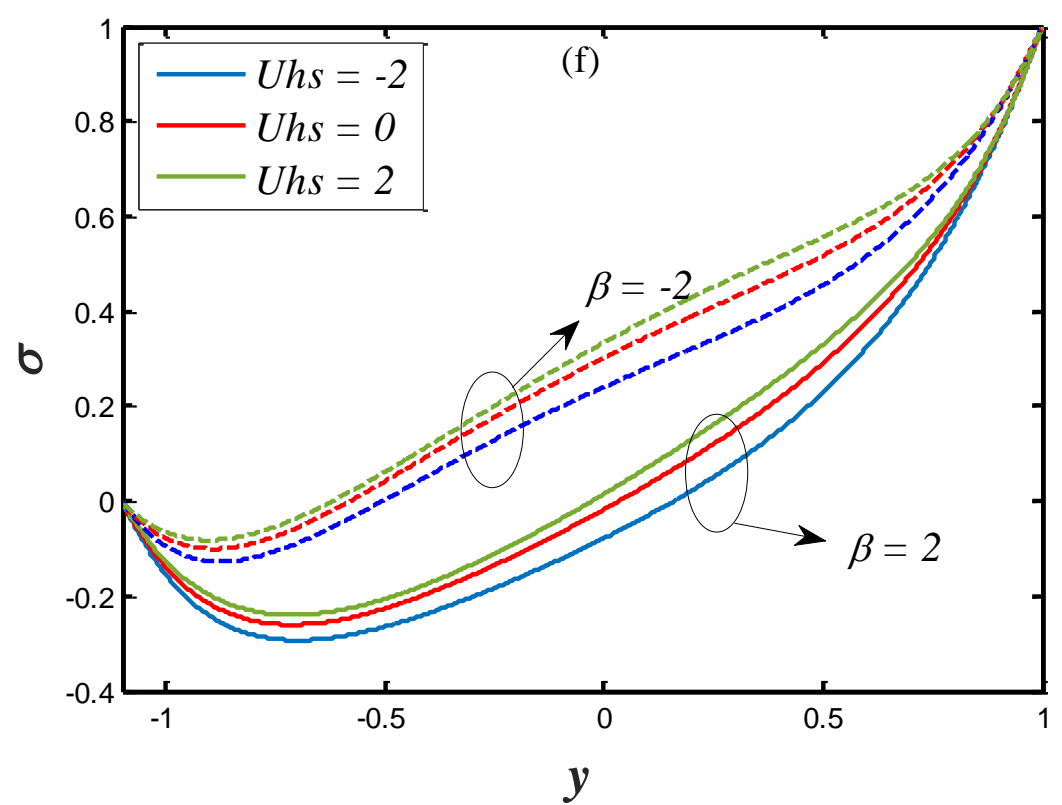

Fig.4.(f). Effect of Helmholtz-Smoluchowski velocity (Uhs) and heat source/sink $(\beta)$ on nanoparticle volume fraction for $a=0.3, b=0.2, \phi=\pi / 4, x=0.5, d=1, \Theta=2, M=2, \kappa=0.2, L=0.3, N t=0.3, N b=0.6, B h_{1}=4, B h_{2}=4, P r$ $=0.71, R n=2, B r=1$.

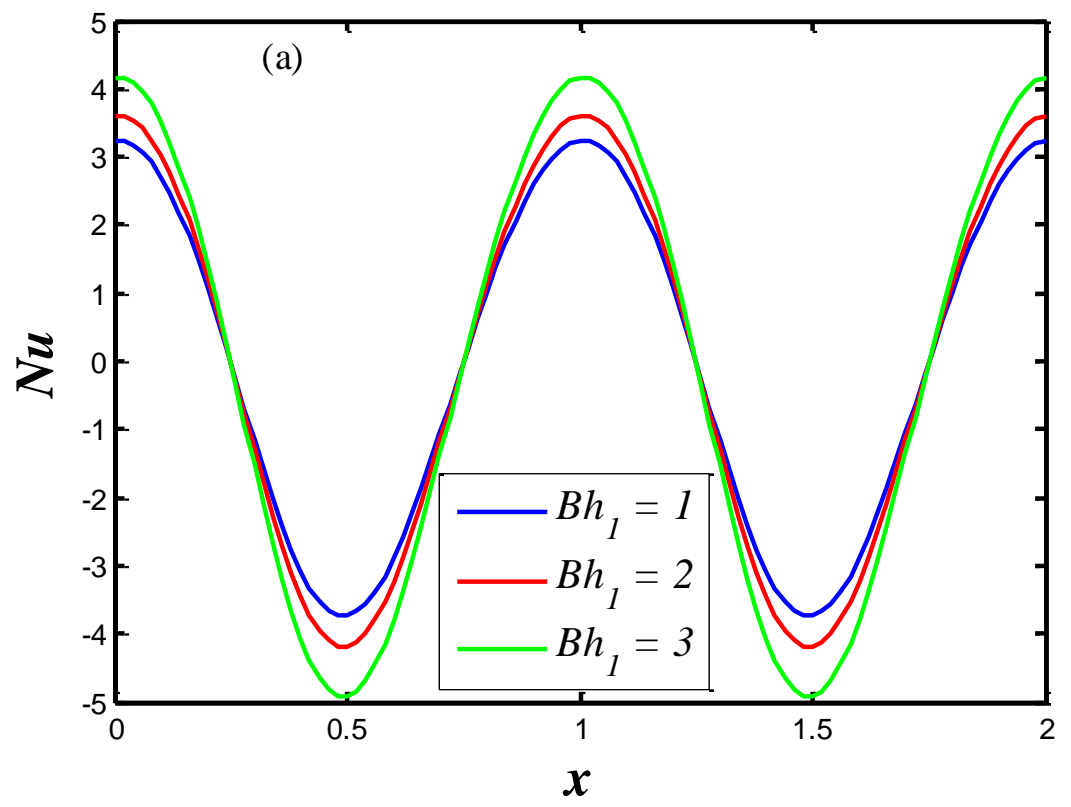

Fig.5.(a). Effect of lower wall thermal Biot number $\left(B h_{1}\right)$ on Nusselt number for $a=0.3, b=0.2, \phi=\pi / 4, d=1, \Theta=1.5$, $\kappa=0.5, L=0.1, N t=0.6, N b=0.6, M=1, B h_{2}=3, \operatorname{Pr}=0.71, R n=0.5, B r=3, \beta=2$, Uhs $=2$. 


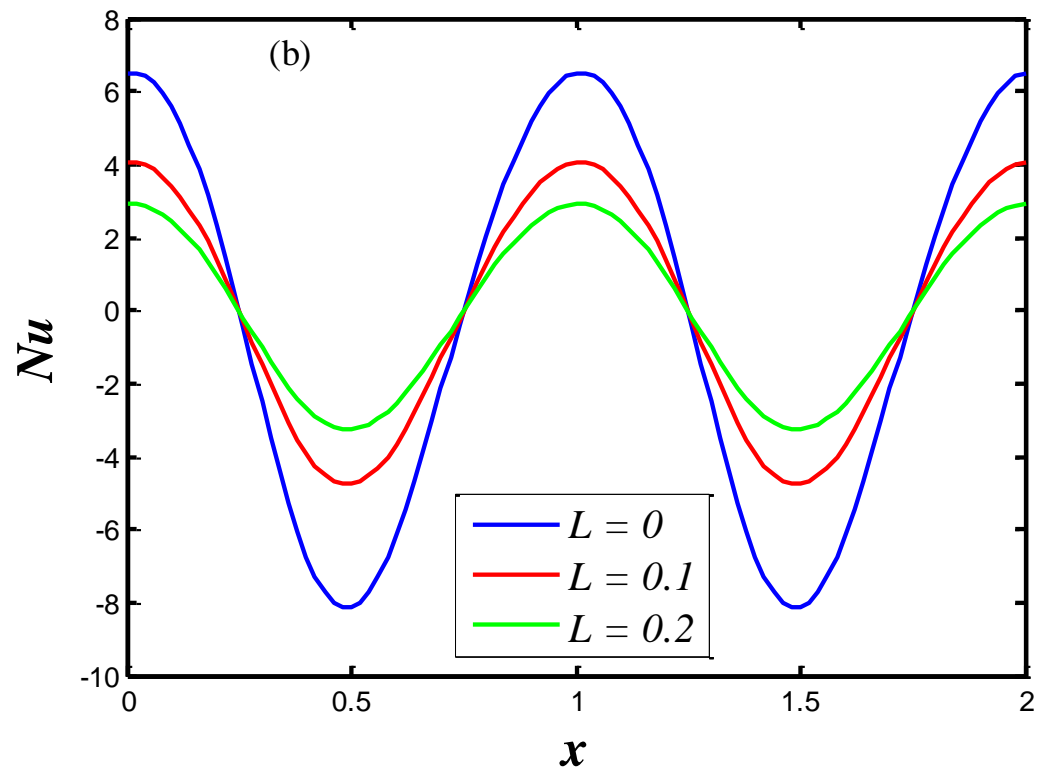

Fig.5.(b). Effect of thermal slip parameter $(L)$ on Nusselt number for $a=0.3, b=0.2, \phi=\pi / 4, d=1, \Theta=1.5, \kappa=0.5$, $N t=0.6, N b=0.6, B h_{1}=3, B h_{2}=3, P r=0.71, R n=0.5, B r=3, \beta=2, M=2$, Uhs $=2$.

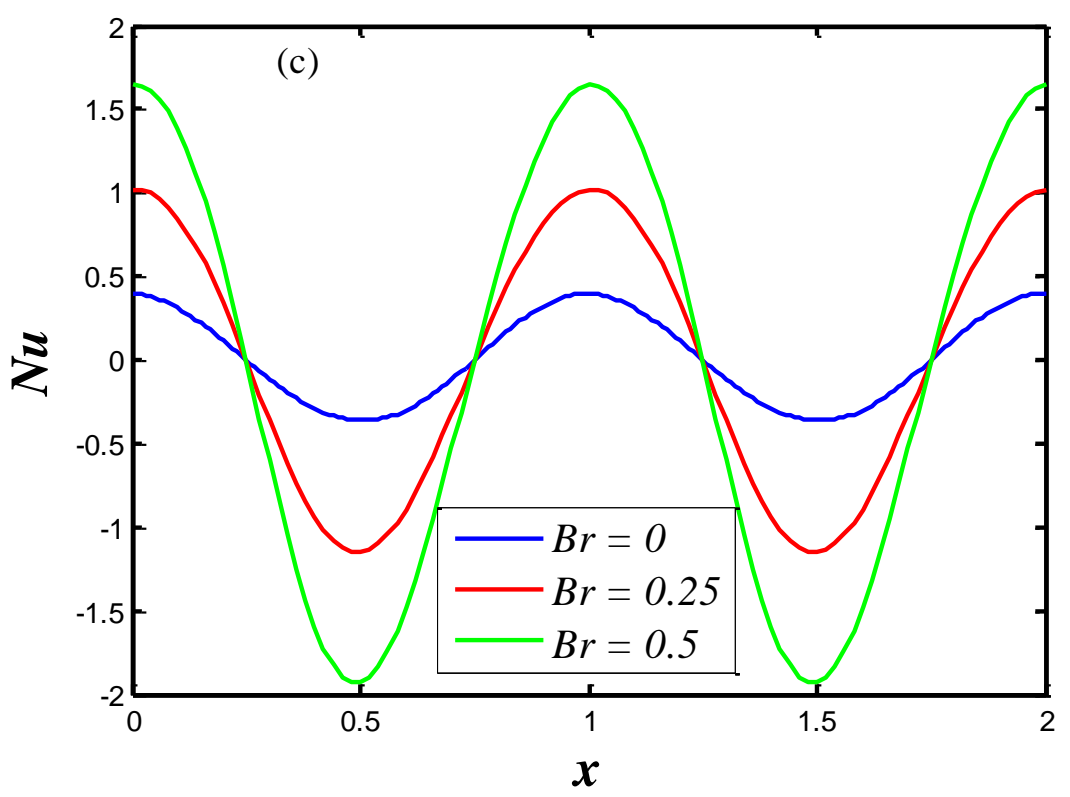

Fig.5.(c). Effect of Brinkman number $(B r)$ on Nusselt number for $a=0.3, b=0.2, \phi=\pi / 4, d=1, \Theta=1.5$, $\kappa=0.5, L=0.3, N t=0.6, N b=0.6, B h_{1}=3, B h_{2}=3, \operatorname{Pr}=0.71, R n=0.5, \beta=2, M=2$, Uhs $=2$. 


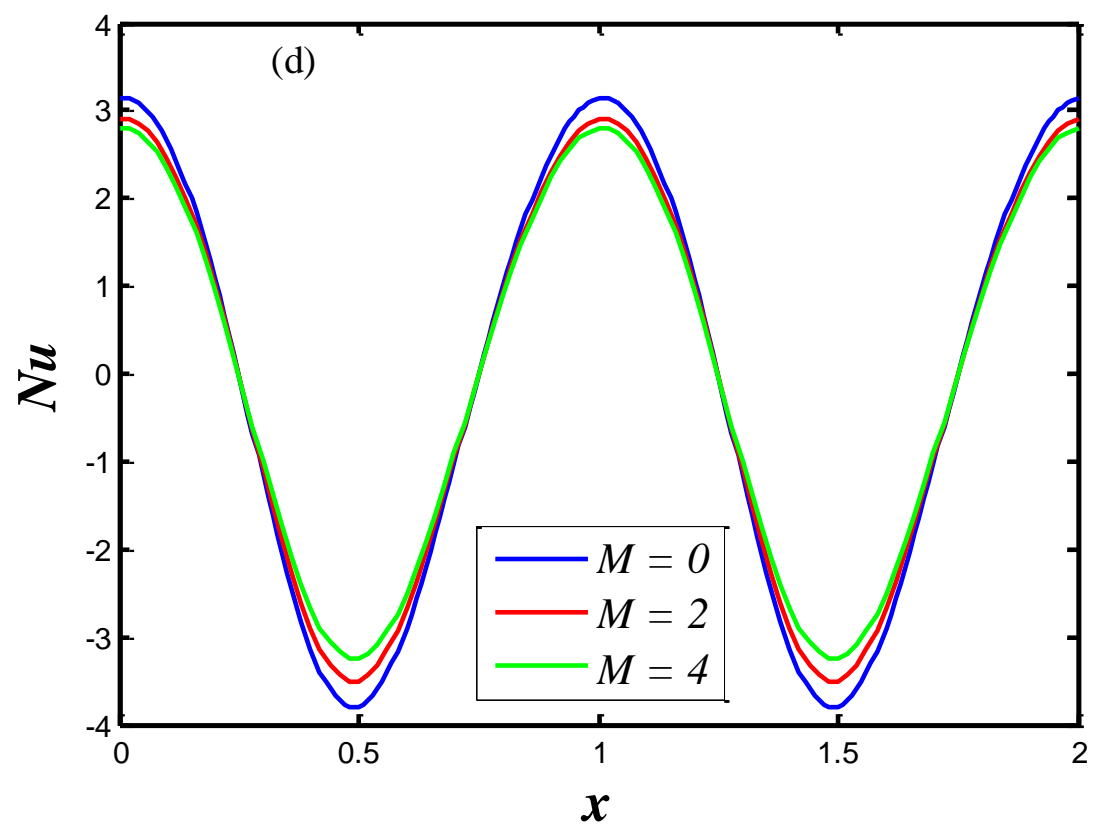

Fig.5.(d). Effect of Hartmann magnetic number $(M)$ on Nusselt number for $a=0.3, b=0.2, \phi=\pi / 4, d=1, \Theta=1.5$, $\kappa=0.5, L=0.3, N t=0.6, N b=0.6, B h_{1}=3, B h_{2}=3, P r=0.71, B r=3 \beta=2, R n=1, U h s=2$.

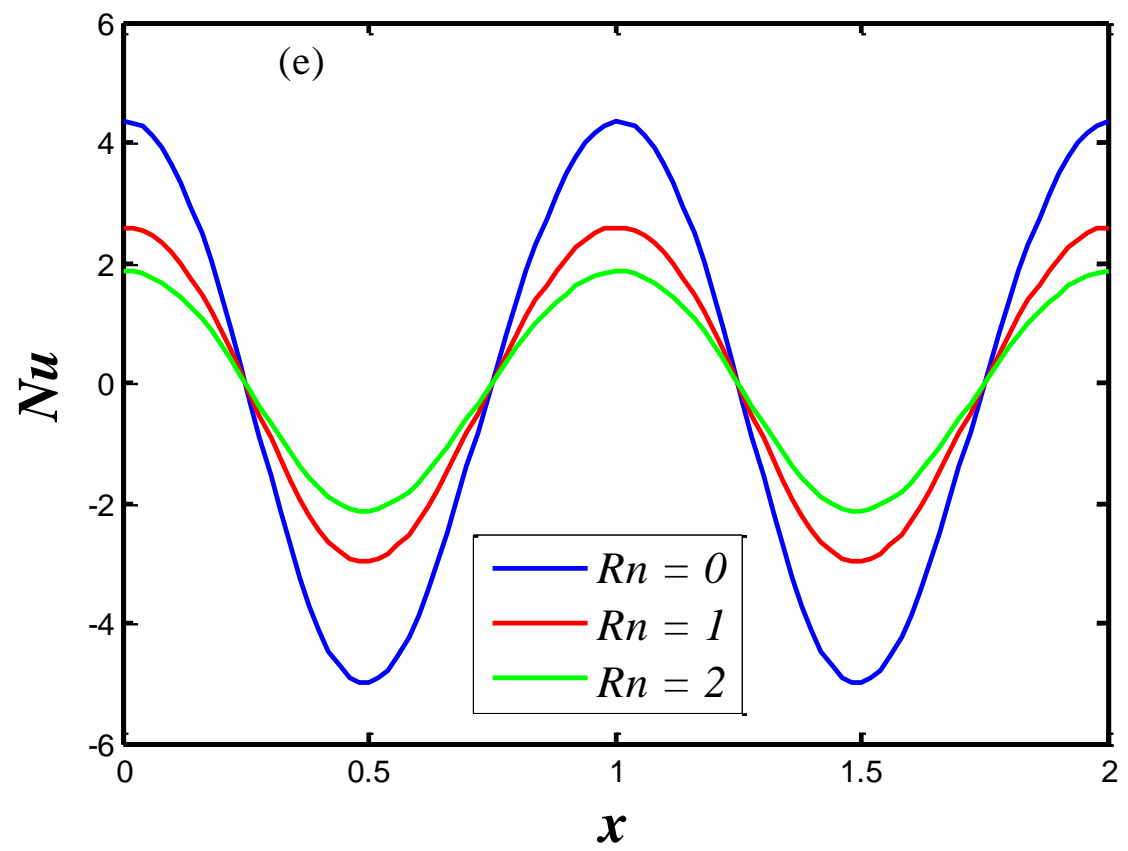

Fig.5.(e). Effect of thermal radiation parameter $(R n)$ on Nusselt number for $a=0.3, b=0.2, \phi=\pi / 4, d=1, \Theta=1.5$, $\kappa=0.5, L=0.3, N t=0.6, N b=0.6, B h_{1}=3, B h_{2}=3, P r=0.71, B r=3, \beta=2, M=2$, Uhs $=2$. 


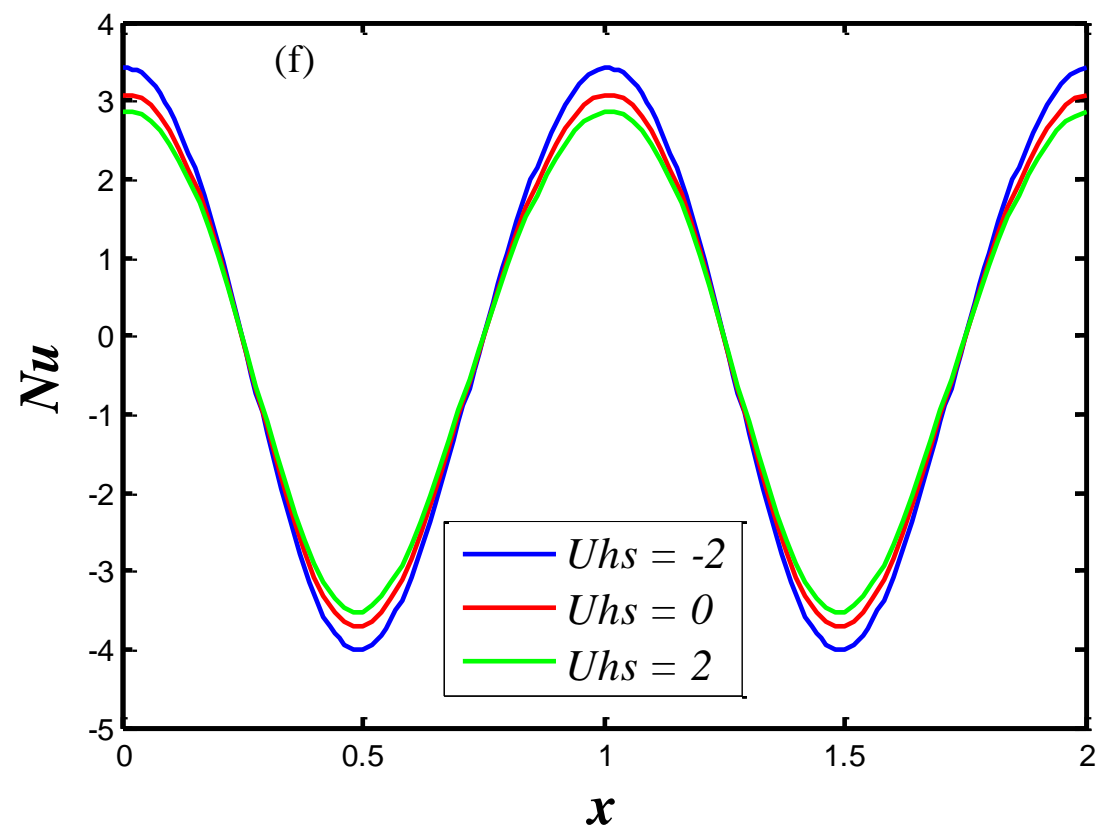

Fig.5.(f). Effect of Helmholtz-Smoluchowski velocity (Uhs) on Nusselt number for $a=0.3, b=0.2, \phi=\pi / 4, d=1$, $\Theta=1.5, \kappa=0.5, L=0.3, N t=0.6, N b=0.6, B h_{1}=3, B h_{2}=3, \operatorname{Pr}=0.71, R n=0.5, \beta=2, B r=3, M=2$.

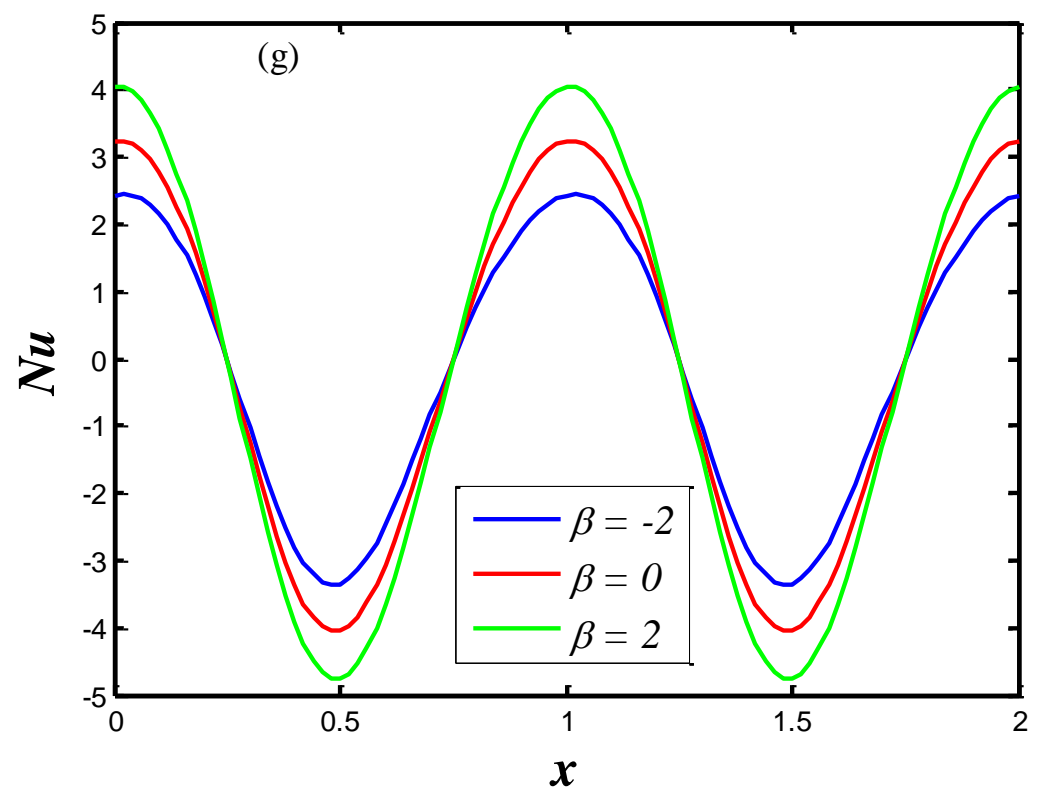

Fig.5.(g). Effect of heat source/sink parameter $(\beta)$ on Nusselt number for $a=0.3, b=0.2, \phi=\pi / 4, d=1, \Theta=1.5$, $\kappa=0.5, L=0.3, N t=0.6, N b=0.6, B h_{1}=3, B h_{2}=3, P r=0.71, R n=0.5, B r=3, \beta=2, M=2, U h s=2$. 